\title{
Electrostatic shielding in plasmas and the physical meaning of the Debye length
}

\author{
G. Livadiotis ${ }^{1} \dagger$ and D. J. McComas ${ }^{1,2}$ \\ ${ }^{1}$ Southwest Research Institute, San Antonio, TX, USA \\ ${ }^{2}$ Department of Physics \& Astronomy, University of Texas at San Antonio, San Antonio, TX, USA
}

(Received 26 August 2013; revised 7 October 2013; accepted 10 October 2013;

first published online 20 January 2014)

This paper examines the electrostatic shielding in plasmas, and resolves inconsistencies about what the Debye length really is. Two different interpretations of the Debye length are currently used: (1) The potential energy approximately equals the thermal energy, and (2) the ratio of the shielded to the unshielded potential drops to $1 / \mathrm{e}$. We examine these two interpretations of the Debye length for equilibrium plasmas described by the Boltzmann distribution, and non-equilibrium plasmas (e.g. space plasmas) described by kappa distributions. We study three dimensionalities of the electrostatic potential: 1-D potential of linear symmetry for planar charge density, 2-D potential of cylindrical symmetry for linear charge density, and 3-D potential of spherical symmetry for a point charge. We resolve critical inconsistencies of the two interpretations, including: independence of the Debye length on the dimensionality; requirement for small charge perturbations that is equivalent to weakly coupled plasmas; correlations between ions and electrons; existence of temperature for nonequilibrium plasmas; and isotropic Debye shielding. We introduce a third Debye length interpretation that naturally emerges from the second statistical moment of the particle position distribution; this is analogous to the kinetic definition of temperature, which is the second statistical moment of the velocity distribution. Finally, we compare the three interpretations, identifying what information is required for theoretical/experimental plasma-physics research: Interpretation 1 applies only to kappa distributions; Interpretation 2 is not restricted to any specific form of the ion/electron distributions, but these forms have to be known; Interpretation 3 needs only the second statistical moment of the positional distribution.

\section{Introduction}

The Debye length is not simply a measuring length unit used in the description and study of plasmas. Instead, it represents the physical scale of the transition from plasma collectivity to individual particle behavior. Thus, it interfaces between the physics of micro and macro scales and its technical definition and detailed attributes are crucial for theoretical and experimental plasma-physics research. But what really is the Debye length and how can it be meaningfully and consistently defined and interpreted?

There is currently no strict definition of the term Debye length; instead particular properties of electrostatic shielding have been used for the 'definition' or 'analytical derivation' of this critical length in plasmas. Here we use the word 'interpretation'

$\dagger$ Email address for correspondence: glivadiotis@swri.edu 
to characterize two fundamentally different ways of using 'Debye length' as found in literature: (1) the distance at which the potential energy from a charge perturbation is equal to the thermal energy (e.g. Kallenrode 2004; Baumjohann and Treumann 2012), and (2) the distance at which the potential energy from a charge perturbation has fallen to 1/e of its unshielded value (e.g. Montgomery and Tidman 1964; Chen 1974). Interpretation 1 is a physical property of Debye shielding; it involves the distance where a sort of equilibrium is established between the 'source' of the shielding that is the restoring electric potential, and the 'sink' of the shielding that is the disturbing thermal energy. In contrast, Interpretation 2 is simply a mathematical property of the shielding and marks the distance where the shielded potential falls to a certain fraction of its unshielded value.

To the best of our knowledge, the substantial differences between the two interpretations of the Debye length have never been reconciled. Furthermore, the mathematical equivalence of the two interpretations occurs only at thermal equilibrium, the state of a system where any flow of heat (thermal conduction, thermal convection, thermal radiation) is in balance. Thus, we ask - can the interpretations somehow be reconciled in general and what is the correct definition for Debye length in plasmas that are not in thermal equilibrium?

While the two interpretations are equivalent at thermal equilibrium, they are different for plasmas out of thermal equilibrium, and the validity of Interpretation 1 is not certain for such plasmas. On the other hand, Interpretation 2 is valid for systems both at and out of thermal equilibrium. This is because, similar to the Boltzmann distribution at thermal equilibrium, the kappa distribution can lead to a linearized Poisson equation with an exponential solution. Therefore, both the interpretations of the Debye length have been used for thermal equilibrium plasmas, but only Interpretation 2 has been used for plasmas out of thermal equilibrium that are described by kappa distributions (Bryant 1996; Rubab and Murtaza 2006; Gougam and Tribeche 2011). Indeed, as we will show in this paper, Interpretation 1 needs to be modified for non-equilibrium systems.

Kappa distributions are based on the solid statistical background of non-extensive statistical mechanics (Livadiotis and McComas 2009, 2013b) and describe systems that are in stationary states but out of thermal equilibrium. Stationary states refer to the distribution function of phase space of a system, meaning that must be - at least temporarily - invariant, even though is not given by the classical Boltzmann-Gibbs distribution of energy or the equivalent Maxwell distribution of velocities. Instead, the distribution function for stationary states out of thermal equilibrium are described by kappa distributions, for which the temperature is well-defined and given by the mean kinetic energy (Livadiotis and McComas 2009, 2010a, 2011b, 2012, 2013b), while the kappa index (that governs these distributions) is a thermodynamic parameter inversely proportional to the correlation between the phase space of any two particles (Livadiotis and McComas 2011b, 2013d).

Space plasmas are examples of weakly coupled plasmas that typically reside in stationary states out of thermal equilibrium. Kappa distributions have been successfully applied in numerous space plasmas, e.g. solar wind (e.g. Chotoo et al. 2000; Mann et al. 2002; Maksimovic et al. 2005; Yoon et al. 2006; Pierrard and Lazar 2010), planetary magnetospheres (e.g. Christon 1987; Collier and Hamilton 1995; Grabbe 2000; Mauk et al. 2004; Schippers et al. 2008; Dialynas et al. 2009; Ogasawara et al. 2013), outer heliosphere and inner heliosheath (e.g. Decker and Krimigis 2003; Decker et al. 2005; Heerikhuisen et al. 2008; Zank et al. 2010; Livadiotis et al. 2011, 2012, 2013), and other general plasma analyses (e.g. Milovanov and Zelenyi 2000, 
2001; Saito et al. 2000; Leubner 2004; Raadu and Shafiq 2007; Hellberg et al. 2009; Livadiotis and McComas 2009, 2010a-c, 2011a, 2011b, 2012, 2013a-e; Tribeche et al. 2009; Le Roux et al. 2010; Eslami et al. 2011; Kourakis et al. 2012; Yoon 2012; Yoon et al. 2012; Bains et al. 2013; Saberian and Esfandyari-Kalejahi 2013).

The purpose of this paper is to resolve the inconsistencies of the two interpretations of the Debye length for both equilibrium and non-equilibrium plasmas. We organize the paper as follows. Section 2 briefly explores the two Debye length interpretations with a simple example of one-dimensional (1-D) symmetry of electric field and potential. In Sec. 3, we solve the Poisson equation of Gauss' law of electrodynamics and show the detailed derivation of the Debye length for three different dimensionalities of the potential: 1-D or linear symmetry for planar charge density, two-dimensional (2-D) or cylindrical symmetry for linear charge density, and three-dimensional (3-D) or spherical symmetry for a point charge. We examine plasmas both at and out thermal equilibrium, and compare the two interpretations of the Debye length for all three dimensionalities. In Sec. 4, we discuss the inconsistencies and assumptions of the Debye length interpretations: (1) Independence of the Debye length on the potential dimensionality; (2) approximation of small charge perturbations; (3) cut-off of the electron density; (4) weakly coupled plasmas; (5) no correlations between ions and electrons; (6) existence of temperature for plasmas out of thermal equilibrium; and (7) isotropic Debye shielding. In Sec. 5, we develop the necessary modifications required to eliminate inconsistencies between the two interpretations. In Sec. 6, we provide the restrictions of each of the two interpretations and develop the concept of yet a third interpretation. Finally, Sec. 7 summarizes the conclusions. Six Appendices support the mathematical formalism used in this paper: Appendix A provides the derivation of the kappa distribution density formulations for ions and electrons that are used in this paper; Appendices B and C solve the Poisson equation for equilibrium and non-equilibrium plasmas, respectively; Appendix D shows the equality of the number of ions in a perturbation and the number of excess electrons in the plasma; Appendix E derives the Debye length for large perturbations using Interpretation 1; and Appendix F derives the particle position moments of the charge distribution in the plasma.

\section{Two interpretations of the Debye length in plasmas}

Even stable plasmas constantly undergo charge perturbations via the thermal motion of their particles. Whenever such a charge perturbation occurs in a plasma, positive and negative free charges respond by moving in opposite directions around the perturbation, producing a shielding effect that preserves the charge quasi-neutrality of the plasma (equidistribution of charge density) at large distances. Hence, the shielding electric potential energy recovers the local plasma's stability and restores its quasineutrality. On the other hand, the thermal motions of particles compete with the potential and make it more difficult for free charges to shield the charge perturbation. While the potential energy is larger than the thermal energy at distances near the charge perturbation, the thermal energy prevails at distances far from it. The specific distance, for which the potential and thermal energies are equal, specifies the first interpretation of the Debye length $\lambda_{\mathrm{D}}$ (Kallenrode 2004).

We demonstrate this interpretation with the simple geometry of 1-D shielding (Fig. 1(a)). A local charge perturbation of ions can be approximated by a planar equidistribution of total charge $\Delta Q=e \Delta N$ and charge density $\sigma=\Delta Q / A=e \Delta N / A$, where $A$ is the area covered by the perturbation charge and $e$ denotes the elementary 


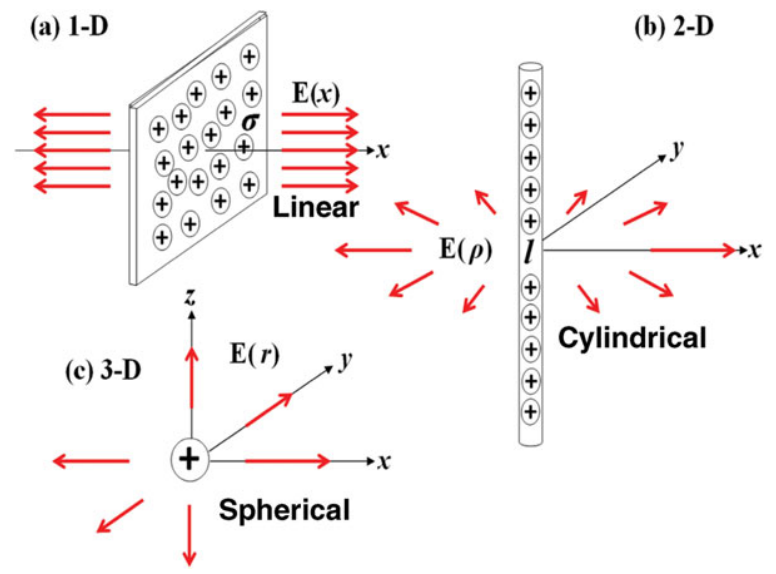

FIGURE 1. (Colour online) Three simple geometries of charge perturbation, with (a) linear, (b) cylindrical, (c) spherical symmetry, and respective dimensionalities $d=1,2,3$. (The electric field notations $\mathbf{E}(x), \mathbf{E}(\rho), \mathbf{E}(r)$ correspond to the surface $\sigma$, linear $l$, and point charge density.)

electric charge. Due to the charge perturbation, a local concentration of charges results in an electric field $E$, with $E=|\mathbf{E}|$, perpendicular to the plane (along the direction of the $x$-axis). Then, (mobile) electrons move along the field, producing an opposite field $-\mathbf{E}$, and restoring the plasma quasi-neutrality.

The surface density of the free charges within a Debye length (from both sides of the plane of the charge perturbation) is $-\sigma=-\Delta N e / A=-2\left(\Delta N / N_{D}\right) e n_{\infty} \lambda_{D}$; a number of $\Delta N$ free electrons is necessary to cancel the perturbation charge (see Appendix D); $-\Delta Q=-e \Delta N$ is the charge of these electrons; $N_{\mathrm{D}}=2 n_{\infty} A \lambda_{\mathrm{D}}$ is the number of ions or electrons in a 'Debye-rod' of cross-sectional area $A$ and length $\lambda_{\mathrm{D}}$; and $n_{\infty}$ denotes the density of the undisturbed plasma at sufficiently large distance for quasi-neutrality to be valid.

The perturbation electric field is $E=\frac{1}{2} \sigma / \varepsilon$ (the fraction $\frac{1}{2}$ comes from the existence of the perturbation electric field $\mathbf{E}$ on both the sides of the plane); $\varepsilon$ is the permittivity of the plasma. The corresponding electric potential energy is $e \Phi(x) \sim e \Phi(0)-$ $\frac{1}{2} e \sigma \varepsilon^{-1} x$ (this is true for small scales $x$ compared to the Debye length, $x \ll \lambda_{\mathrm{D}}$ ). At the Debye length $\lambda_{\mathrm{D}}$, the shielding cancels the charge perturbation, so that $\Phi\left(\lambda_{\mathrm{D}}\right)=0$ or $\Phi(0) \sim \frac{1}{2} \sigma \varepsilon^{-1} \lambda_{\mathrm{D}}$. On the other hand, the kinetic energy per particle is $\sim \frac{1}{2} k_{\mathrm{B}} T_{0}$ ( $\frac{1}{2}$ comes from one degree of freedom), where $T_{0} \equiv\left(T_{i}^{-1}+T_{e}^{-1}\right)^{-1}$ is the 'effective' temperature, which includes both the ion $T_{i}$ and electron $T_{e}$ temperature. Hence, the ratio of the per particle potential to thermal energy is given by

$$
\frac{e \Phi(x)}{\frac{1}{2} k_{\mathrm{B}} T_{0}} \cong \frac{\sigma}{e n_{\infty} \lambda_{\mathrm{D}}} \cdot \frac{\frac{1}{2} e^{2} n_{\infty} \varepsilon^{-1} \lambda_{\mathrm{D}}^{2}}{\frac{1}{2} k_{\mathrm{B}} T_{0}} \cdot\left[\frac{\Phi(0)}{\frac{1}{2} \sigma \varepsilon^{-1} \lambda_{\mathrm{D}}}-\frac{x}{\lambda_{\mathrm{D}}}\right],
$$

and noting that $\Phi(0) \sim \frac{1}{2} \sigma \varepsilon^{-1} \lambda_{\mathrm{D}}$ and $\sigma /\left(e n_{\infty} \lambda_{\mathrm{D}}\right)=2 \Delta N / N_{\mathrm{D}}$, we obtain

$$
\frac{e \Phi(x)}{\frac{1}{2} k_{\mathrm{B}} T_{0}} \cong 2 \frac{\Delta N}{N_{\mathrm{D}}} \cdot \frac{\frac{1}{2} e^{2} n_{\infty} \varepsilon^{-1} \lambda_{\mathrm{D}}^{2}}{\frac{1}{2} k_{\mathrm{B}} T_{0}} \cdot\left(1-\frac{x}{\lambda_{\mathrm{D}}}\right) .
$$

The potential energy is due to the presence of $\Delta N$ ions that contribute to the charge perturbation. On the other hand, the thermal energy applies to all the $N_{D}$ particles that could be available to shield the charge perturbation, i.e. included in a Debye length. Hence, the ratio, $R$, of the potential to thermal energy must be normalized by 
the quantity $\Delta N / N_{\mathrm{D}}$, i.e.

$$
R \equiv \frac{N_{\mathrm{D}} e \Phi}{\Delta N \frac{1}{2} k_{\mathrm{B}} T_{0}}
$$

that is,

$$
R(x) \cong 2 \cdot \frac{e^{2} \varepsilon^{-1} \lambda_{\mathrm{D}}^{2}}{k_{\mathrm{B}} T_{0}} \cdot\left(1-x / \lambda_{\mathrm{D}}\right) .
$$

The functional part of $(3 \mathrm{a}),\left(1-x / \lambda_{\mathrm{D}}\right)$, is valid for $x \ll \lambda_{\mathrm{D}}$, and is generally given by the exponential $\exp \left(-x / \lambda_{\mathrm{D}}\right)$, i.e.

$$
R(x) \cong 2 \cdot \frac{e^{2} \varepsilon^{-1} \lambda_{\mathrm{D}}^{2}}{k_{\mathrm{B}} T_{0}} \cdot \exp \left(-x / \lambda_{\mathrm{D}}\right)
$$

In Appendix B.1, we derive (3b) by solving the Poisson equation. There we show that

$$
R(x) \cong 2 \exp \left(-x / \lambda_{\mathrm{D}}\right) \text {. }
$$

Comparing (3b) and (3c) we find that the Debye length $\lambda_{\mathrm{D}}$ is given by

$$
\lambda_{\mathrm{D}} \equiv \sqrt{\frac{k_{\mathrm{B}} \varepsilon}{e^{2}} \frac{T_{0}}{n_{\infty}}} .
$$

The case described above is characterized as 1-D, because the planar charge perturbation of surface density $\sigma$ leads to an electric potential/field of linear symmetry, $\Phi(x)$ and $\mathbf{E}(x)$ (Appendix B.1). Moreover, in this paper we also examine the simple examples of 2-D and 3-D geometries, as shown in Figs 1(b) and (c). In the 2-D case, a charge perturbation of linear density $l$ leads to an electric potential/field of cylindrical symmetry, $\Phi(\rho)$ and $\mathbf{E}(\rho)$ (Appendix B.2), while in the 3-D case, a pointcharge perturbation leads to an electric potential/field of spherical symmetry, $\Phi(r)$ and $\mathbf{E}(r)$, (Appendix B.3; $\rho$ and $r$ are the cylindrical and spherical radii, respectively).

The ratio of the total potential to thermal energy at the Debye length is a constant of the order of unity. Indeed, in the 1-D case, we have $R\left(x=\lambda_{\mathrm{D}}\right) \cong 1 / \mathrm{e}$. In addition, in Sec. 3 we show that for any dimensionality $d$ of the potential, we have

$$
\text { (Interpretation1) } R\left(\text { distance } \sim \lambda_{\mathrm{D}}\right) \cong C(d) \cdot(1 / e) \sim O(1),
$$

where the constant $C(d)$ differs for each dimensionality $d=1,2,3$, but remains in the order of unity. This constitutes Interpretation 1 of the Debye length: The ratio $R$ of the potential to the thermal energy is some constant of the order of unity. Figure 2(a) demonstrates this interpretation of Debye length through (5a) that is the approximate equality of the potential energy by the thermal energy at this distance.

An alternative and very frequently used interpretation of the Debye length (e.g. Montgomery and Tidman 1964; Chen 1974) involves the distance where the exponential factor, which is included in the spatial function of the electric potential, falls to $\sim 1 / \mathrm{e}$ of its unshielded value. For the 1-D case, the spatial dependence of the potential is given only by the exponential factor, i.e. $\Phi(x) \sim \Phi(0) \cdot \exp \left(-x / \lambda_{\mathrm{D}}\right)$, so that $\Phi\left(\lambda_{\mathrm{D}}\right) \sim \Phi(0) /$ e. In the 2-D and 3-D cases, the potential follows similar radial behavior, $\Phi(\rho) \propto \exp \left(-\rho / \lambda_{\mathrm{D}}\right) / \sqrt{\rho}$ and $\Phi(r) \propto \exp \left(-r / \lambda_{\mathrm{D}}\right) / r$. The deviation from the unshielded (us) potential, $\Phi_{\text {us }}(\rho) \propto 1 / \sqrt{\rho}$ and $\Phi_{\text {us }}(r) \propto 1 / r$, comes from the exponential factor that falls to $\sim 1 / \mathrm{e}$ of its value at distance equal to $\lambda_{\mathrm{D}}$, defining thus, the Debye length in the 2-D and 3-D potentials. Therefore, this interpretation could be written in a compact way for any $d$-dimensional $(d$-D) potential,

$$
\text { (Interpretation 2) } \Phi\left(\lambda_{\mathrm{D}}\right) / \Phi_{\mathrm{us}} \cong 1 / \mathrm{e} \sim O(1) .
$$




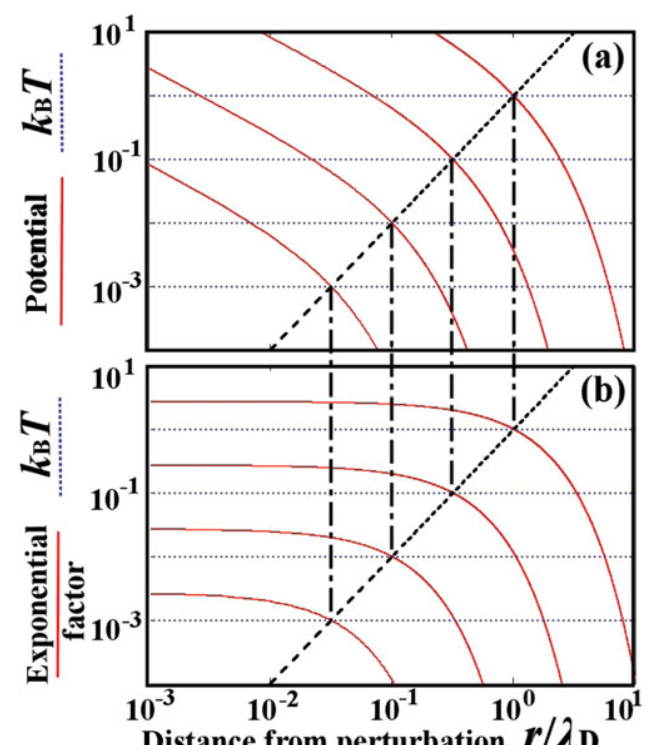

Figure 2. (Colour online) Demonstration of Interpretations 1 and 2 of the Debye length in plasmas. (a) Interpretation 1: The 3-D potential energy at thermal equilibrium (Appendix B, (B18a), (B18b)) (red) and the thermal energy (blue) are co-plotted for four values of thermal energy, $k_{\mathrm{B}} T / \mathrm{J}=10^{-3}, 10^{-2}, 10^{-1}, 1$ (corresponding to four values of the potential energy because the potential's formulation involves also the temperature). The potential energy $e \Phi$ is multiplied by $3 \mathrm{e} N / \Delta N$ in order to be exactly equal to the thermal energy $k_{\mathrm{B}} T$ at a distance equal to the Debye length. (b) Interpretation 2: The exponential factor $\exp \left(-r / \sqrt{k_{\mathrm{B}} T}\right)$ (red) and the thermal energy (blue) are co-plotted for the same four values of thermal energy. Note any involved parameter other than the temperature is normalized to 1 . The exponential factor is multiplied by $k_{\mathrm{B}} T / \mathrm{e}$ in order to be exactly equal to the thermal energy $k_{\mathrm{B}} T$ at a distance equal to the Debye length. In both panels, the geometric locus of the intersections (connected with vertical dash-dot lines) is given by the parabola $k_{\mathrm{B}} T=\lambda_{\mathrm{D}}^{2}$ (on a $\log -\log$ scale).

This constitutes Interpretation 2 of the Debye length, demonstrated in Fig. 2(b).

The two Debye length interpretations, namely (i) via the competing thermal and potential energies and their ratio, (5a), and (ii) via the exponential form of the potential, (5b), are physically different, but they result to the same Debye length, thus they are mathematically equivalent at thermal equilibrium. However, for plasmas out of thermal equilibrium, the two interpretations do not necessarily result to the same Debye length, and (5a) and (5b) are not equivalent for plasmas out of thermal equilibrium. As we will see, these need to be modified for kappa distributions.

Once, the specific formulation of the phase space distribution function is known, the Poisson equation for Gauss' law in electrodynamics can be solved to derive the exact potential configuration. At thermal equilibrium, the phase space distribution is given by the Boltzmann-Maxwell distribution, while plasmas in stationary states out of thermal equilibrium are typically described by the kappa distribution. In order to proceed from equilibrium to the non-equilibrium plasmas, it is critical to understand the concept of temperature for systems in stationary states that are out of thermal equilibrium. Recently the temperature was shown to be well-defined for these nonequilibrium systems described by kappa distributions (for details, see Livadiotis and McComas 2009, 2010a, 2011b, 2013b; see also the early work of Treumann 1999; Treumann et al. 2004; Treumann and Jaroschek 2008). Sections 4.6 and 4.7 show in detail the connection of temperature with the Debye length. 
According to Interpretations 1 and 2, (5a) and (5b), the Debye length is given by (4) for plasmas at thermal equilibrium. However, using Interpretation 2, the Debye length is modified to $\lambda_{\mathrm{D}, \mathrm{K}}$ for plasmas out of thermal equilibrium that are described by kappa distributions (Bryant 1996; Rubab and Murtaza 2006; Gougam and Tribeche 2011; see also Sec. 4),

$$
\lambda_{\mathrm{D}, \mathrm{K}} \equiv \lambda_{\mathrm{D} \infty} \cdot \mathrm{K}\left(\kappa_{0}\right), \operatorname{withK}\left(\kappa_{0}\right) \equiv \sqrt{\frac{\kappa_{0}}{\kappa_{0}+1}}=\sqrt{\frac{\kappa-\frac{3}{2}}{\kappa-\frac{1}{2}}}, \lambda_{\mathrm{D} \infty} \equiv \sqrt{\frac{k_{\mathrm{B}} \varepsilon}{e^{2}} \frac{T_{0}}{n_{\infty}}} .
$$

Note that we use the invariant notation of the universal kappa index, $\kappa_{0}$, that is independent of the dimensionality $d$, in contrast to the usual kappa index that depends on $d$, i.e. $\kappa=\kappa_{0}+d / 2$ (Livadiotis and McComas 2011b).

Hereafter, we use the notation $\lambda_{\mathrm{D}}$ for the actual Debye length, whatever that may be. A second subscript is added to indicate if the Debye length is given by (i) the form for plasmas at thermal equilibrium, $\lambda_{\mathrm{D}}=\lambda_{\mathrm{D} \infty}$, (ii) the form for plasmas out of thermal equilibrium and described by kappa distributions, $\lambda_{\mathrm{D}}=\lambda_{\mathrm{D}, \mathrm{K}}$, or (iii) some other form, $\lambda_{\mathrm{D}}=\lambda_{\mathrm{D}, \Lambda}$, which is given in terms of some kappa function, $\Lambda\left(\kappa_{0}\right)$,

$$
\lambda_{\mathrm{D}, \Lambda} \equiv \lambda_{\mathrm{D} \infty} \cdot \Lambda\left(\kappa_{0}\right) .
$$

If $\lambda_{\mathrm{D}}=\lambda_{\mathrm{D} \infty}$ is correct, then $\Lambda\left(\kappa_{0}\right)=1$. If $\lambda_{\mathrm{D}}=\lambda_{\mathrm{D}, \mathrm{K}}$ is correct, then $\Lambda\left(\kappa_{0}\right)=\mathrm{K}\left(\kappa_{0}\right)$. It might be that the correct Debye length is different from either of these cases, corresponding to some different function $\Lambda\left(\kappa_{0}\right)$. Next, we derive the Debye length for plasmas at thermal equilibrium and verify that it is given by $\lambda_{\mathrm{D}}=\lambda_{\mathrm{D} \infty}$ using both Interpretations 1 and 2. Then, we examine the Debye length for plasmas out of thermal equilibrium, and determine whether $\lambda_{\mathrm{D}}$ is given by $\lambda_{\mathrm{D} \infty}, \lambda_{\mathrm{D}, \mathrm{K}}$, or some other $\lambda_{\mathrm{D}, \Lambda}$.

\section{Debye length in equilibrium and non-equilibrium plasmas}

\subsection{Poisson equation}

For plasmas at thermal equilibrium, the ion/electron densities are given by the Boltzmann distribution of energy,

$$
n_{i}=n_{\infty} \cdot \exp \left[-\frac{e \Phi(\mathbf{r})}{k_{\mathrm{B}} T_{i}}\right], n_{e}=n_{\infty} \cdot \exp \left[\frac{e \Phi(\mathbf{r})}{k_{\mathrm{B}} T_{e}}\right],
$$

where $n_{\infty}$ denotes again the ion or electron density. For plasmas in stationary states out of thermal equilibrium, the ion and electron densities are described by kappa distributions, expressed in terms of the invariant kappa index $\kappa_{0}$. This is invariant under variations of the system's particles $N$ and the total degrees of freedom $f_{N}=d \cdot N$, where $d$ denotes now the kinetic degrees of freedom per particle. (For details on the derivations, see Appendices; for more details on the kappa distribution formulation, see Livadiotis and McComas 2011b, 2013b.) Namely,

$$
n_{i}=n_{\infty} \cdot\left[1+\frac{e \Phi(\mathbf{r})}{\kappa_{0} k_{\mathrm{B}} T_{i}-e \bar{\Phi}_{i}}\right]^{-\kappa_{0}-1}, n_{e}=n_{\infty} \cdot\left[1-\frac{e \Phi(\mathbf{r})}{\kappa_{0} k_{\mathrm{B}} T_{e}+e \bar{\Phi}_{e}}\right]^{-\kappa_{0}-1},
$$

where $\bar{\Phi}$ is the average potential energy.

The total charge density, $\rho=e\left(n_{i}-n_{e}\right)$, is expanded in terms of the ratio of the potential to the thermal energy. More precisely, for the equilibrium case, it is expanded assuming that $e \Phi(\mathbf{r}) /\left(k_{\mathrm{B}} T_{0}\right) \ll 1$, while for the non-equilibrium case, it is expanded 


$\begin{array}{llll}\text { Charge dimension } & \text { Charge distribution } & \text { Potential/field dimension } & \text { Potential/field symmetry } \\ 2 & \text { Surface density } \sigma & 1 & \text { Linear }(x) \\ 1 & \text { Linear density } l & 2 & \text { Cylindrical }(\rho) \\ 0 & \text { Point charge } Q & 3 & \text { Spherical }(r)\end{array}$

TABLE 1. Charge and potential/field dimensions and symmetry.

\begin{tabular}{|c|c|c|}
\hline$d$-D & $e \Phi / k_{\mathrm{B}} T_{0}$ & $R=\left(N_{\mathrm{D}} / \Delta N\right) \cdot\left(e \Phi / \frac{1}{2} k_{\mathrm{B}} T_{0}\right)$ \\
\hline \multicolumn{3}{|c|}{ Equilibrium } \\
\hline & $(1 / 1) \cdot \frac{\sigma}{2 e n_{\infty} \lambda_{D_{\infty}}} \cdot \exp \left(-x / \lambda_{D_{\infty}}\right)$ & $(2 / 1) \cdot \exp \left(-x / \lambda_{D_{\infty}}\right)$ \\
\hline 2 & $(1 / \sqrt{\pi}) \cdot \frac{l}{e n_{\infty} \pi \lambda_{D_{\infty}}^{2}} \cdot \frac{\exp \left(-\rho / \lambda_{D_{\infty}}\right)}{\sqrt{\rho / \lambda_{D_{\infty}}}}$ & $(2 / \sqrt{\pi}) \cdot \frac{\exp \left(-\rho / \lambda_{\mathrm{D} \infty}\right)}{\sqrt{\rho / \lambda_{\mathrm{D} \infty}}}$ \\
\hline 3 & $(1 / 3) \cdot \frac{Q}{e n_{\infty}(4 \pi / 3) \lambda_{D_{\infty}}^{3}} \cdot \frac{\exp \left(-r / \lambda_{D_{\infty}}\right)}{r / \lambda_{\infty}}$ & $(2 / 3) \cdot \frac{\exp \left(-r / \lambda_{D_{\infty}}\right)}{r / \lambda_{D_{\infty}}}$ \\
\hline & {$\left[d \Gamma\left(\frac{d+1}{2}\right)\right]^{-1} \cdot \frac{\Delta N}{N_{D}} \cdot \frac{\exp \left(-r / \lambda_{D_{\infty}}\right)}{\left(r / \lambda_{D_{\infty}}\right)^{(d-1) / 2}}$} & {$\left[\frac{d}{2} \Gamma\left(\frac{d+1}{2}\right)\right]^{-1} \cdot \frac{\exp \left(-r / \lambda_{D_{\infty}}\right)}{\left(r / \lambda_{D_{\infty}}\right)^{(d-1) / 2}}$} \\
\hline \multicolumn{3}{|c|}{ Non-equilibrium } \\
\hline & $(1 / 1) \cdot \frac{\sigma}{2 e n_{\infty} \lambda_{D_{\infty}}} \cdot\left(\frac{\kappa_{0}}{\kappa_{0}+1}\right)^{1 / 2} \cdot \exp \left(-x / \lambda_{D, \mathrm{~K}}\right)$ & $(2 / 1) \cdot \Lambda\left(\kappa_{0}\right)\left(\frac{\kappa_{0}}{\kappa_{0}+1}\right)^{1 / 2} \cdot \exp \left(-x / \lambda_{D, \mathrm{~K}}\right)$ \\
\hline 2 & $(1 / \sqrt{\pi}) \cdot \frac{l}{e n_{\infty} \pi \lambda_{D_{\infty}}^{2}} \cdot \frac{\exp \left(-\rho / \lambda_{D, K}\right)}{\sqrt{\rho / \lambda_{D_{\mathrm{K}}}}}$ & $(2 / \sqrt{\pi}) \cdot \Lambda\left(\kappa_{0}\right)^{2} \cdot \frac{\exp \left(-\rho / \lambda_{D, \mathrm{~K}}\right)}{\sqrt{\rho / \lambda_{D_{\mathrm{K}}}}}$ \\
\hline 3 & $(1 / 3) \cdot \frac{Q}{e n_{\infty}(4 \pi / 3) \lambda_{D \propto}^{3}} \cdot\left(\frac{\kappa_{0}}{\kappa_{0}+1}\right)^{-1 / 2} \cdot \frac{\exp \left(-r / \lambda_{D, \mathrm{~K}}\right)}{r / \lambda_{D, \mathrm{~K}}}$ & $(2 / 3) \cdot \Lambda\left(\kappa_{0}\right)^{3}\left(\frac{\kappa_{0}}{\kappa_{0}+1}\right)^{-1 / 2} \cdot \frac{\exp \left(-r / \lambda_{D, \mathrm{~K}}\right)}{r / \lambda_{D, \mathrm{~K}}}$ \\
\hline$d$ & $\begin{array}{l}{\left[d \Gamma\left(\frac{d+1}{2}\right)\right]^{-1} \cdot \frac{\Delta N}{N_{D}} \cdot \Lambda\left(\kappa_{0}\right)^{d}\left(\frac{\kappa_{0}}{\kappa_{0}+1}\right)^{(2-d) / 2}} \\
\quad \cdot \frac{\exp \left(-r / \lambda_{D, \mathrm{~K}}\right)}{\left(r / \lambda_{D, \mathrm{~K}}\right)^{(d-1) / 2}}\end{array}$ & $\begin{array}{l}{\left[\frac{d}{2} \Gamma\left(\frac{d+1}{2}\right)\right]^{-1} \cdot \Lambda\left(\kappa_{0}\right)^{d}\left(\frac{\kappa_{0}}{\kappa_{0}+1}\right)^{(2-d) / 2}} \\
\quad \cdot \frac{\exp \left(-r / \lambda_{, \mathrm{K}}\right)}{\left(r / \lambda_{D, \mathrm{~K}}\right)^{(d-1) / 2}}\end{array}$ \\
\hline
\end{tabular}

TABLE 2. Potential energy and ratio of potential to thermal energy for the three dimensionalities.

assuming that $e \Phi(\mathbf{r}) /\left(\frac{\kappa_{0}}{\kappa_{0}+1} k_{\mathrm{B}} T_{0}\right) \ll 1$. Hence, we obtain

$$
\rho(\mathbf{r}) / \varepsilon \cong-\frac{e^{2} n_{\infty}}{k_{B} \varepsilon}\left(\frac{1}{T_{i}}+\frac{1}{T_{e}}\right) \cdot \Phi(\mathbf{r})=-\frac{1}{\lambda_{\mathrm{D} \infty}^{2}} \cdot \Phi(\mathbf{r})
$$

for plasmas at thermal equilibrium, and

$$
\rho(r) / \varepsilon \cong-\frac{e^{2} n_{\infty}}{k_{\mathrm{B}} \varepsilon}\left(\frac{1}{T_{i}}+\frac{1}{T_{e}}\right) \cdot \frac{\kappa_{0}+1}{\kappa_{0}} \cdot \Phi(r)=-\frac{1}{\lambda_{\mathrm{D} \infty}^{2}} \cdot \frac{\kappa_{0}+1}{\kappa_{0}} \cdot \Phi(r)=-\frac{1}{\lambda_{\mathrm{D}, \mathrm{K}}^{2}} \cdot \Phi(r)
$$

for plasmas out of thermal equilibrium. Then, the linearized Poisson equation is given by

$$
\nabla^{2} \Phi(\mathbf{r})=-\rho(\mathbf{r}) / \varepsilon \cong \frac{1}{\lambda_{\mathrm{D}, \mathrm{K}}^{2}} \cdot \Phi(\mathbf{r}) .
$$

This is solved for both equilibrium and non-equilibrium cases (Appendices B and $\mathrm{C}$, respectively), by considering the three dimensionalities of potential/electric field, as given in Table 1 and illustrated in Fig. 1.

\subsection{Results}

Table 2 gathers the derived formulations of the potential for all three dimensionalities, i.e. 1-D ( $\Phi$ and $\mathbf{E}$ with linear symmetry along $x$-axis, for planar charge density on $y$ - $z$ plane), 2-D ( $\Phi$ and $\mathbf{E}$ with cylindrical symmetry on $x-y$ plane, for linear charge density 


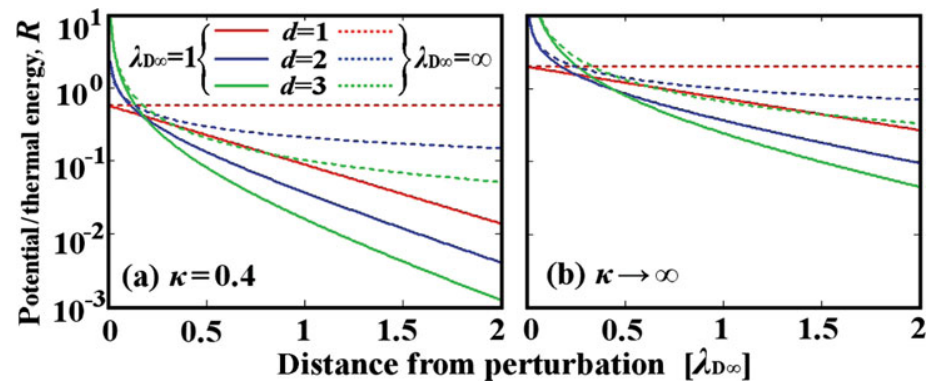

Figure 3. (Colour online) Functional behavior of the ratio of the potential to thermal energy. This is depicted for the three dimensionalities $d=1,2,3$ (Table 2), for (a) $\kappa_{0}=0.4$, and (b) $\kappa_{0} \rightarrow \infty$, considering that $\Lambda\left(\kappa_{0}\right)=\sqrt{\frac{\kappa_{0}}{\kappa_{0}+1}}$. The ratio is compactly written for any $d$, i.e. $R(\xi) \cong C(d) \cdot\left(\frac{\kappa_{0}}{\kappa_{0}+1}\right)^{(3+d) / 4} \xi^{(1-d) / 2} \exp \left(-\sqrt{\frac{\kappa_{0}+1}{\kappa_{0}}} \xi\right)$, with $C(d) \equiv\left[\frac{d}{2} \Gamma\left(\frac{d+1}{2}\right)\right]^{-1}$ and distant from perturbation $\xi \equiv r / \lambda_{\mathrm{D} \infty}$. The unshielded case $\left(\lambda_{\mathrm{D} \infty} \rightarrow \infty\right)$ is also shown for each dimensionality (dash lines), for reference. We observe that for large distances compared to the Debye length, the shielding is more effective for $d=3$, while for quite smaller distances, the shielding is more effective for $d=1$.

on $z$-axis), and 3-D ( $\Phi$ and $\mathbf{E}$ with spherical symmetry, for a point charge). For each, the potential energy is normalized to the thermal energy, i.e. $e \Phi / k_{B} T_{0}$. The similarities between the three cases are apparent. We observe that for all three dimensionalities the potential is expressed in terms of the electron excess $\Delta N$ normalized to the Debye number of particles $N_{D}$, i.e.

$$
\begin{aligned}
\frac{\Delta N}{N_{D}}(d=1) & =\frac{\sigma}{2 e n_{\infty} \lambda_{D, \Lambda}}, \frac{\Delta N}{N_{D}}(d=2)=\frac{l}{e n_{\infty} \pi \lambda_{D, \Lambda}^{2}}, \frac{\Delta N}{N_{D}}(d=3) \\
& =\frac{Q}{e n_{\infty}(4 \pi / 3) \lambda_{D, \Lambda}^{3}},
\end{aligned}
$$

where $\lambda_{\mathrm{D}, \Lambda}$ is reduced to $\lambda_{\mathrm{D} \infty}$ at thermal equilibrium. The right column of Table 2 gives the ratio of the potential to thermal energy, $R$. This is shown in Fig. 3 for the equilibrium and non-equilibrium cases, and all three dimensionalities $d=1,2,3$.

\subsection{Comparing the two interpretations}

Table 3 compares the results for the two interpretations of the Debye length, given in (5a) and (5b), for both equilibrium and non-equilibrium plasmas, and for all three dimensionalities $d=1,2,3$. We observe that the two interpretations of the Debye length are different for the equilibrium and non-equilibrium cases.

Interpretation 2 , that is $\Phi\left(\lambda_{\mathrm{D}, \Lambda}\right) / \Phi_{\mathrm{us}}=1 / \mathrm{e}$ as stated in $(5 \mathrm{~b})$, is consistent for both the equilibrium and non-equilibrium cases when $\lambda_{\mathrm{D}, \Lambda}=\lambda_{\mathrm{D}, \mathrm{K}}$, i.e. $\Lambda\left(\kappa_{0}\right)=\sqrt{\frac{\kappa_{0}}{\kappa_{0}+1}}$. Then, the shielded potential falls to $1 / \mathrm{e}$ of its unshielded value at a distance equal to the Debye length, independently of the kappa index. However, the 1/e threshold is arbitrary, and there is no substantial physical reason for this to be $\sim 1 / \mathrm{e}$ rather than some other value of the order of unity. Further, this may even be some function of the kappa index and not a constant. For a given function of $\Lambda\left(\kappa_{0}\right)$, different than $\sqrt{\frac{\kappa_{0}}{\kappa_{0}+1}}$, the threshold becomes a function of $\kappa_{0}$, given by $\exp \left[-\Lambda\left(\kappa_{0}\right) \sqrt{\frac{\kappa_{0}+1}{\kappa_{0}}}\right] \equiv G\left(\kappa_{0}\right)$ (as given in Table 3). As an example, the threshold is modeled by the $\kappa$-deformed exponential function $\exp _{\mathrm{K}}(x) \equiv\left(1-\frac{1}{\kappa_{0}} x\right)^{-\kappa_{0}-1}$ (called also $q$-exponential from the entropic index $q_{0}=1+1 / \kappa_{0}$; see also, appendix $\mathrm{A}$ in Livadiotis and McComas 
$d-\mathrm{D} \quad\left(\right.$ i) $R\left(\lambda_{\mathrm{D}, \Lambda}\right)$

Equilibrium

$1 \quad(2 / 1) \cdot(1 / \mathrm{e})$

$2 \quad(2 / \sqrt{\pi}) \cdot(1 / \mathrm{e})$

$3 \quad(2 / 3) \cdot(1 / \mathrm{e})$

$d \quad\left[\frac{d}{2} \Gamma\left(\frac{d+1}{2}\right)\right]^{-1} \cdot(1 / \mathrm{e})$

Non-equilibrium

$1 \quad(2 / 1) \cdot\left(\lambda_{\mathrm{D}, \Lambda} \lambda_{\mathrm{D}, \mathrm{K}} / \lambda_{\mathrm{D} \infty}^{2}\right) \cdot \exp \left(-\lambda_{\mathrm{D}, \Lambda} / \lambda_{\mathrm{D}, \mathrm{K}}\right)=$

$(2 / 1) \cdot \Lambda\left(\kappa_{0}\right) \sqrt{\frac{\kappa_{0}}{\kappa_{0}+1}} \cdot \exp \left[-\Lambda\left(\kappa_{0}\right) \sqrt{\frac{\kappa_{0}+1}{\kappa_{0}}}\right]$

$2(2 / \sqrt{\pi}) \cdot\left(\lambda_{\mathrm{D}, \Lambda}^{3 / 2} \lambda_{\mathrm{D}, \mathrm{K}}^{1 / 2} / \lambda_{\mathrm{D} \infty}^{2}\right) \cdot \exp \left(-\lambda_{\mathrm{D}, \Lambda} / \lambda_{\mathrm{D}, \mathrm{K}}\right)=$

$(2 / \sqrt{\pi}) \cdot \Lambda\left(\kappa_{0}\right)^{3 / 2}\left(\frac{\kappa_{0}}{\kappa_{0}+1}\right)^{1 / 4} \cdot \exp \left[-\Lambda\left(\kappa_{0}\right) \sqrt{\frac{\kappa_{0}+1}{\kappa_{0}}}\right]$

$3 \quad(2 / 3) \cdot\left(\lambda_{\mathrm{D}, \Lambda}^{2} / \lambda_{\mathrm{D} \infty}^{2}\right) \cdot \exp \left(-\lambda_{\mathrm{D}, \Lambda} / \lambda_{\mathrm{D}, \mathrm{K}}\right)=$

$(2 / 3) \cdot \Lambda\left(\kappa_{0}\right)^{2} \cdot \exp \left[-\Lambda\left(\kappa_{0}\right) \sqrt{\frac{\kappa_{0}+1}{\kappa_{0}}}\right]$

$d \quad\left[\frac{d}{2} \Gamma\left(\frac{d+1}{2}\right)\right]^{-1} \cdot\left[\lambda_{\mathrm{D}, \Lambda}^{(d+1) / 2} \lambda_{\mathrm{D}, \mathrm{K}}^{(3-d) / 2} / \lambda_{\mathrm{D} \infty}^{2}\right] \cdot \exp \left(-\lambda_{\mathrm{D}, \Lambda} / \lambda_{\mathrm{D}, \mathrm{K}}\right)=$

$\left[\frac{d}{2} \Gamma\left(\frac{d+1}{2}\right)\right]^{-1} \cdot \Lambda\left(\kappa_{0}\right)^{(d+1) / 2} \cdot\left(\frac{\kappa_{0}}{\kappa_{0}+1}\right)^{(3-d) / 4} \cdot \exp \left[-\Lambda\left(\kappa_{0}\right) \sqrt{\frac{\kappa_{0}+1}{\kappa_{0}}}\right]$

Non-equilibrium, $\Lambda\left(\kappa_{0}\right)=\sqrt{\frac{\kappa_{0}}{\kappa_{0}+1}}$

$d \quad\left[\frac{d}{2} \Gamma\left(\frac{d+1}{2}\right)\right]^{-1} \cdot(1 / \mathrm{e}) \cdot\left(\frac{\kappa_{0}}{\kappa_{0}+1}\right)$

TABLE 3. Two interpretations of the Debye length. (ii) $\Phi\left(\lambda_{\mathrm{D}, \Lambda}\right) / \Phi_{\mathrm{us}}$

$1 / \mathrm{e}$
$1 / \mathrm{e}$
$1 / \mathrm{e}$
$1 / \mathrm{e}$

$\exp \left(-\lambda_{\mathrm{D}, \Lambda} / \lambda_{\mathrm{D}, \mathrm{K}}\right)=$

$\exp \left[-\Lambda\left(\kappa_{0}\right) \sqrt{\frac{\kappa_{0}+1}{\kappa_{0}}}\right]$

$\exp \left(-\lambda_{\mathrm{D}, \Lambda} / \lambda_{\mathrm{D}, \mathrm{K}}\right)=$

$\exp \left[-\Lambda\left(\kappa_{0}\right) \sqrt{\frac{\kappa_{0}+1}{\kappa_{0}}}\right]$

$\exp \left(-\lambda_{\mathrm{D}, \Lambda} / \lambda_{\mathrm{D}, \mathrm{K}}\right)=$

$\exp \left[-\Lambda\left(\kappa_{0}\right) \sqrt{\frac{\kappa_{0}+1}{\kappa_{0}}}\right]$

$\exp \left(-\lambda_{\mathrm{D}, \Lambda} / \lambda_{\mathrm{D}, \mathrm{K}}\right)=$

$\exp \left[-\Lambda\left(\kappa_{0}\right) \sqrt{\frac{\kappa_{0}+1}{\kappa_{0}}}\right]$

$1 / \mathrm{e}$

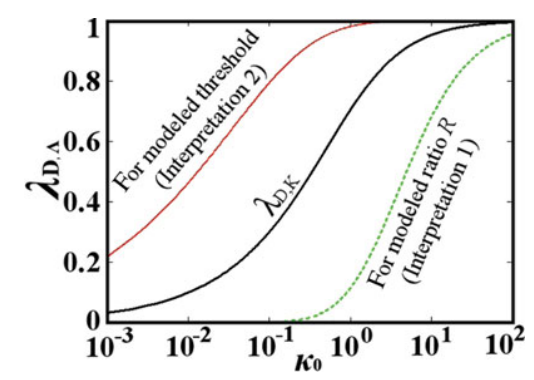

FIGURE 4. (Colour online) Functional behavior of the Debye length $\lambda_{\mathrm{D}, \Lambda}$ on the kappa index (denoted as $\Lambda\left(\kappa_{0}\right)$ ). For the modified Debye length $\lambda_{\mathrm{D}, \Lambda} \sim \lambda_{\mathrm{D}, \mathrm{K}}$, it is $\Lambda\left(\kappa_{0}\right) \sim \sqrt{\frac{\kappa_{0}}{\kappa_{0}+1}}$ (black solid). This is consistent with both Interpretation $1, R\left(\lambda_{\mathrm{D}, \Lambda}\right)=C(d) \cdot \frac{\kappa_{0}}{\kappa_{0}+1}$, and Interpretation 2, $\Phi\left(\lambda_{\mathrm{D}, \Lambda}\right) / \Phi_{\mathrm{us}}=1 / \mathrm{e}$. Deviations from the behavior of $\Lambda\left(\kappa_{0}\right) \sim \sqrt{\frac{\kappa_{0}}{\kappa_{0}+1}}$ may exist when these equalities do not hold. For example, we derive $\Lambda\left(\kappa_{0}\right)$ via Interpretation 1 using $R\left(\lambda_{\mathrm{D}, \Lambda}\right)=C(d) \cdot c \cdot\left[\kappa_{0} /\left(\kappa_{0}+1\right)\right]^{a}$, with $c \cong 0.3679, d=3, a=5$ (green dash), and via Interpretation 2 using $\Phi\left(\lambda_{\mathrm{D}, \Lambda}\right) / \Phi_{\mathrm{us}}=\exp _{\mathrm{K}}(-1)$ (red solid).

2009), and thus, $\exp _{\mathrm{K}}(-1)=\left(\frac{\kappa_{0}}{\kappa_{0}+1}\right)^{\kappa_{0}+1}$ replaces $\exp (-1)=1 / \mathrm{e}$. Then, we derive $\Lambda\left(\kappa_{0}\right)=\sqrt{\kappa_{0}\left(\kappa_{0}+1\right)} \ln \left(1+\frac{1}{\kappa_{0}}\right)$, as shown in Fig. 4.

An important characteristic of Interpretation 1 is that the ratio $R\left(\lambda_{\mathrm{D}, \Lambda}\right)$ differs for the three dimensionalities $d=1,2,3$, while for Interpretation 2 , the ratio $\Phi\left(\lambda_{\mathrm{D}, \Lambda}\right) / \Phi_{\mathrm{us}}$ is identical for all three dimensionalities. For thermal equilibrium plasmas, we have $R\left(\lambda_{\mathrm{D} \infty}\right) \cong C(d) \cdot(1 / e)$, where $C(d) \equiv\left[\frac{d}{2} \Gamma\left(\frac{d+1}{2}\right)\right]^{-1}$. For non-equilibrium plasmas, we 
have that the ratio $R$ is a different function of $\kappa_{0}$ for each $d$, i.e.

$$
\begin{aligned}
& R\left(\lambda_{D, \Lambda} ; d\right)=C(d) \cdot \frac{\kappa_{0}}{\kappa_{0}+1} \cdot\left[\Lambda\left(\kappa_{0}\right) / \sqrt{\frac{\kappa_{0}}{\kappa_{0}+1}}\right]^{\frac{1+d}{2}} \cdot \exp \left[-\Lambda\left(\kappa_{0}\right) / \sqrt{\frac{\kappa_{0}}{\kappa_{0}+1}}\right], \\
& C(d) \equiv\left[\frac{d}{2} \Gamma\left(\frac{d+1}{2}\right)\right]^{-1}, d=1,2,3 .
\end{aligned}
$$

The only function $\Lambda\left(\kappa_{0}\right)$ for which the three dimensionalities become equivalent is $\Lambda\left(\kappa_{0}\right)=\sqrt{\frac{\kappa_{0}}{\kappa_{0}+1}}$, that is the modified Debye length, $\lambda_{\mathrm{D}, \mathrm{K}},(6)$. Then, the ratio of the potential to thermal energy at $\lambda_{\mathrm{D}, \mathrm{K}}$ becomes

$$
R\left(\lambda_{\mathrm{D}, \mathrm{K}} ; d\right)=C(d) \cdot\left(\frac{\kappa_{0}}{\kappa_{0}+1}\right) \cdot(1 / \mathrm{e}), d=1,2,3 .
$$

In contrast to Interpretation 2, which depends on some arbitrary threshold of the decreasing potential with distance, Interpretation 1 is based on a physical relationship, the distance for which the potential and thermal energies are comparable. According to Interpretation 1 in (5a), for plasmas at thermal equilibrium the ratio $R$ is a constant of the order of unity. However, as we observe in (12a), for plasmas out of thermal equilibrium this is not true, but the ratio $R$ is some function of $\kappa_{0}$. When $\Lambda\left(\kappa_{0}\right)=\sqrt{\frac{\kappa_{0}}{\kappa_{0}+1}},(12 \mathrm{a})$ is reduced to $(12 \mathrm{~b})$; then, the ratio $R$ has much simpler form, but still depends on $\kappa_{0}$. Nevertheless, the quantity $C(d) \cdot\left(\frac{\kappa_{0}}{\kappa_{0}+1}\right) \cdot(1 / \mathrm{e})$ is still of the order of unity for large kappa indices. In particular, for the near-equilibrium region, which is determined for $\kappa_{0}>1$ (Livadiotis and McComas 2010, 2011, 2013d), we have $R\left(\lambda_{\mathrm{D}, \mathrm{K}} ; d\right) \sim O(1)$. However, the ratio $R$ cannot be of the order of unity for the far-equilibrium region, which is determined for $\kappa_{0} \ll 1$, because then $R\left(\lambda_{\mathrm{D}, \mathrm{K}} ; d\right) \ll 1$.

In general, different forms of $R\left(\lambda_{\mathrm{D}, \Lambda} ; \kappa_{0}\right)$ may lead to different Debye length forms, i.e. with different dependence on $\kappa_{0}$. This is derived by equalizing the modeled functional form of $R\left(\lambda_{\mathrm{D}, \Lambda} ; \kappa_{0}\right)$ with the respective function given in Table 3 . For example, assume that $R\left(\lambda_{\mathrm{D}, \Lambda} ; \kappa_{0}\right)$ is modeled by the functional form $R\left(\lambda_{\mathrm{D}, \Lambda} ; \kappa_{0}\right)=$ $C(d) \cdot c \cdot\left[\kappa_{0} /\left(\kappa_{0}+1\right)\right]^{a}$, with $c \cong 0.3679$ so that $\Lambda\left(\kappa_{0} \rightarrow \infty\right) \rightarrow 1$. This is equalized to $\left[\frac{d}{2} \Gamma\left(\frac{d+1}{2}\right)\right]^{-1} \cdot \Lambda\left(\kappa_{0}\right)^{(d+1) / 2} \cdot\left(\frac{\kappa_{0}}{\kappa_{0}+1}\right)^{(3-d) / 4} \cdot \exp \left[-\Lambda\left(\kappa_{0}\right) \sqrt{\frac{\kappa_{0}+1}{\kappa_{0}}}\right]$, from which we extract the form of $\Lambda\left(\kappa_{0}\right)$. Figure 4 shows the derived function of $\Lambda\left(\kappa_{0}\right)$ for $a=5$.

\section{Issues and inconsistencies}

The results of the previous section suggest that the Debye length is characterized by the known dependence on the kappa index, $\sim \sqrt{\frac{\kappa_{0}}{\kappa_{0}+1}}$, independently of the dimensionality of the potential $d$. Namely, $\Lambda\left(\kappa_{0}\right) \sim \sqrt{\frac{\kappa_{0}}{\kappa_{0}+1}}$, or $\lambda_{\mathrm{D}, \Lambda} \sim \lambda_{\mathrm{D}, \mathrm{K}}$, for any $d=1,2,3$. However, the two interpretations of the Debye length are interwoven with several critical inconsistencies and considerations that are discussed separately below.

\subsection{Independence of the Debye length on the dimensionality}

A fundamental question is whether the Debye length depends on the geometry of the perturbation charge distribution. If it does, then the Debye length must also depend on the dimensionality $d$. Interpretation 2 does not support such dependence. Indeed, as shown in Table 3 , the ratio $\Phi\left(\lambda_{\mathrm{D}, \Lambda}\right) / \Phi_{\mathrm{us}}$ is identical for any $d$ (for both the equilibrium and non-equilibrium cases). However, Interpretation 1 may lead to a Debye length that depends on the dimensionality $d$. For the specific case of $R\left(\lambda_{\mathrm{D}, \Lambda}\right)=C(d) \cdot(1 / \mathrm{e}) \cdot \frac{\kappa_{0}}{\kappa_{0}+1}$, 


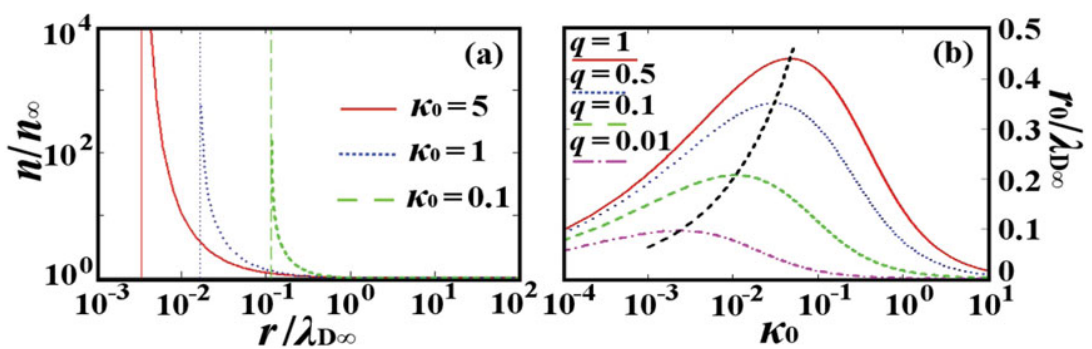

FIGURE 5. (Colour online) (a) Electron density is depicted as a function of $r / \lambda_{\mathrm{D} \infty}$, for $\kappa_{0}=0.1$, $1,5, q \equiv Q /\left[e n_{\infty}(4 \pi / 3) \lambda_{\mathrm{D} \infty}^{3}\right]=0.1, T_{e}=T_{i}$, and dimensionality $d=3$. We observe the cutoff condition at $r=r_{0}$. (b) Plot of the cut-off distance $r_{0}$ as a function of the kappa index $\kappa_{0}$, for $T_{e}=T_{i}$ and $q=0.01,0.1,0.5,1$. The maximum of $r_{0} / \lambda_{\mathrm{D} \infty}$ follows the geometric locus of $2 \sqrt{\kappa_{0}\left(\kappa_{0}+1\right)}$ (indicated with dash line).

$C(d) \equiv\left[\frac{d}{2} \Gamma\left(\frac{d+1}{2}\right)\right]^{-1}$, we obtain $\Lambda\left(\kappa_{0}\right)=\sqrt{\frac{\kappa_{0}}{\kappa_{0}+1}}$, independently of $d$, (12a) and (12b). In general, however, the ratio $R\left(\lambda_{\mathrm{D}, \Lambda}\right)$ may be some function of $\kappa_{0}$ other than $\sim \frac{\kappa_{0}}{\kappa_{0}+1}$. Then, the Debye length would have a dependence on $\kappa_{0}$ different than $\sim \sqrt{\frac{\kappa_{0}}{\kappa_{0}+1}}$ that might vary with the dimensionality $d$. For example, considering the modeled function $R\left(\lambda_{\mathrm{D}, \Lambda}\right) \sim c \cdot\left[\kappa_{0} /\left(\kappa_{0}+1\right)\right]^{a}$ (Sec. 3.3), we find $\Lambda\left(\kappa_{0}\right)$ that depends on $d$. Also, considering the more complicated dependence of $R\left(\lambda_{\mathrm{D}, \Lambda}\right)$ on $\kappa_{0}$ that is originated by large charge perturbations (Appendix E), we find $\Lambda\left(\kappa_{0}\right) \sim \frac{d+1}{2} \cdot \sqrt{\frac{\kappa_{0}}{\kappa_{0}+1}}$.

\subsection{Small charge perturbation approximation}

The derivation of the Debye length in plasmas is attained under the assumption of small charge perturbations, $e \Phi(r) \ll \frac{\kappa_{0}}{\kappa_{0}+1} k_{\mathrm{B}} T_{i, e}$ (Appendix C, (C5)), so that the Poisson equation can be linearized. Note that the perturbation is compared with the temperature and the kappa index, $\kappa_{0}$. Thus, even for small potential energy $e \Phi(r)$, the perturbation may not be small if the temperature and/or the kappa index are also small and tend toward zero. For large charge perturbations, the Debye length cannot be trivially determined from the nonlinear Poisson equation and may not even be a meaningfully defined term. In Appendix E, we find that the Debye length can behave like $\lambda_{\mathrm{D}, \Lambda} \sim \lambda_{\mathrm{D}, \mathrm{K}}$, or, $\Lambda\left(\kappa_{0}\right) \sim \sqrt{\frac{\kappa_{0}}{\kappa_{0}+1}}$, even for large charge perturbations. Nevertheless, the problem can be avoided if the Debye length is restricted to small charge perturbations. Then, Interpretation 1 must be modified to be valid only for small charge perturbations (see Sec. 5).

\subsection{Cut-off of the electron and ion densities}

The formulation of the electron density, shown in (8b), is ill-defined in the case where the quantity $\kappa_{0} k_{B} T_{e}-e \Phi(\mathbf{r})$ becomes negative. In Fig. 5(a) we plot the electron density $n_{e}(r) / n_{\infty}$ (for $d=3$ ), showing that for small distances $r$ from the perturbation the density diverges and cannot be defined. The plot is depicted as a function of $r / \lambda_{\mathrm{D} \infty}$, and for several kappa indices $\kappa_{0}$. The cut-off distance for which the density peaks can be shown that is given by $r_{0} / \lambda_{\mathrm{D} \infty} \cong t_{i} \cdot q$, where $t_{i} \equiv T_{i} /\left(T_{i}+T_{e}\right), q \equiv Q /\left[e n_{\infty}(4 \pi / 3) \lambda_{\mathrm{D} \infty}^{3}\right] . r_{0}$ is the minimum distance for which the potential is well-defined via the electron density of (8b). Figure 5(b) plots this distance $r_{0}$ with respect to $\kappa_{0}$, and various values of $q$.

In reality, both the ion and electron densities can be ill-defined. Indeed, in (8a) we observe that the electron density is ill-defined when $\kappa_{0} k_{B} T_{e}-e \Phi(\mathbf{r})+e \bar{\Phi}_{e}<0$, while the ion density is ill-defined when $\kappa_{0} k_{B} T_{i}+e \Phi(\mathbf{r})-e \bar{\Phi}_{i}<0$. The origin of these inconsistencies is that the kappa distribution function of the phase space has 
been integrated over all the possible velocities, from zero to infinity (for each velocity component). In the following, we reconstruct the electron density to be well-defined even for small values of the potential energy. In order to accomplish this, we integrate the distribution function of the phase space, so that the velocities are restricted by the cut-off energy. The one-particle kappa distribution, defined in the six-dimensional phase space spanned by $(\mathbf{r}, \mathbf{u})$, is given in the case of electrons by

$$
P\left(\mathbf{r}, \mathbf{u} ; \kappa_{0}, T_{e}\right) \sim\left[1+\frac{1}{\kappa_{0}} \cdot \frac{H(\mathbf{r}, \mathbf{u})}{k_{B} T_{e}}\right]^{-\kappa_{0}-\frac{5}{2}},
$$

(where we consider $\mathbf{u}_{b} \equiv<\mathbf{u}>=0$ for simplicity). The Hamiltonian is given in terms of a zero-mean or 'centric' potential energy $\Phi_{\mathrm{C}}(\mathbf{r}) \equiv \Phi(\mathbf{r})-\bar{\Phi}_{e}$, i.e. $H(\mathbf{r}, \mathbf{u})=$ $\frac{1}{2} m \mathbf{u}^{2}-e \Phi_{\mathrm{C}}(\mathbf{r})$. This is a case of negative potential energy (Livadiotis and McComas 2009, 2013b) and the Hamiltonian is not always positive; still, the quantity $1+$ $H(\mathbf{r}, \mathbf{u}) /\left(\kappa_{0} k_{B} T_{e}\right)$ in (13) must be always non-negative (because it represents the kinetic energy $\varepsilon$ ), hence, $\varepsilon \equiv \frac{1}{2} m \mathbf{u}^{2} \geqslant e \Phi_{\mathrm{C}}(\mathbf{r})-\kappa_{0} k_{B} T_{e}$. If the quantity at the right-hand side of this inequality is positive, it gives the smallest possible kinetic energy, $\varepsilon_{\mathrm{M}}(\mathbf{r}) \equiv$ $e \Phi_{\mathrm{C}}(\mathbf{r})-\kappa_{0} k_{B} T_{e}$. If this quantity is negative, then the kinetic energy has no lower restriction and $\varepsilon_{\mathrm{M}}$ is zero. Namely, $\varepsilon_{\mathrm{M}}(\mathbf{r})=e \Phi_{\mathrm{C}}(\mathbf{r})-\kappa_{0} k_{B} T_{e}$ if $e \Phi_{\mathrm{C}}(\mathbf{r})-\kappa_{0} k_{\mathrm{B}} T_{e} \geqslant 0$, and $\varepsilon_{\mathrm{M}}(\mathbf{r})=0$ if $e \Phi_{\mathrm{C}}(\mathbf{r})-\kappa_{0} k_{\mathrm{B}} T_{e} \leqslant 0$. The Tsallis cut-off condition (Tsallis 2009) may be used to express both the cases, i.e. $\varepsilon_{\mathrm{M}}(\mathbf{r})=\left[e \Phi_{\mathrm{C}}(\mathbf{r})-\kappa_{0} k_{B} T_{e}\right]_{+}$, where we utilized the operator $[x]_{+}=x$ if $x \geqslant 0$ and $[x]_{+}=0$ if $x \leqslant 0$. The integration over the velocities leads to the density dependence on the potential and the position $\mathbf{r}$, i.e.

$$
n_{e}\left(\mathbf{r} ; \kappa_{0}, T_{e}\right)=n_{\infty} \cdot \frac{\int_{\varepsilon \operatorname{Me}(\mathbf{r})}^{\infty}\left[1+\frac{\varepsilon-e \Phi(\mathbf{r})}{\kappa_{0} k_{B} T_{e}+e \bar{\Phi}_{e}}\right]^{-\kappa_{0}-\frac{5}{2}} \varepsilon^{\frac{1}{2}} d \varepsilon}{\int_{\varepsilon \operatorname{Me}(\mathbf{r})}^{\infty}\left(1+\frac{\varepsilon}{\kappa_{0} k_{B} T_{e}+e \bar{\Phi}_{e}}\right)^{-\kappa_{0}-\frac{5}{2}} \varepsilon^{\frac{1}{2}} d \varepsilon},
$$

where $\varepsilon_{\mathrm{Me}}(\mathbf{r})=\left[e \Phi(\mathbf{r})-e \bar{\Phi}_{e}-\kappa_{0} k_{B} T_{e}\right]_{+}$. Following the same steps for the density of ions, we obtain

$$
n_{i}\left(\mathbf{r} ; \kappa_{0}, T_{i}\right)=n_{\infty} \cdot \frac{\int_{\varepsilon M i(\mathbf{r})}^{\infty}\left[1+\frac{\varepsilon+e \Phi(\mathbf{r})}{\kappa_{0} k_{B} T_{i}-e \bar{\Phi}_{e}}\right]^{-\kappa_{0}-\frac{5}{2}} \varepsilon^{\frac{1}{2}} d \varepsilon}{\int_{\varepsilon M i(\mathbf{r})}^{\infty}\left(1+\frac{\varepsilon}{\kappa_{0} k_{B} T_{i}-e \bar{\Phi}_{e}}\right)^{-\kappa_{0}-\frac{5}{2}} \varepsilon^{\frac{1}{2}} d \varepsilon},
$$

where $\varepsilon_{\mathrm{Mi}}(\mathbf{r})=\left[-e \Phi(\mathbf{r})+e \bar{\Phi}_{i}-\kappa_{0} k_{B} T_{i}\right]_{+}$. Using a step function, $\Theta(x>0)=1$ and $\Theta(x<0)=0,(14 \mathrm{a})$ and $(14 \mathrm{~b})$ become

$$
\begin{gathered}
n_{e}\left(\mathbf{r} ; \kappa_{0}, T_{e}\right)=n_{\infty} \cdot \frac{\int_{0}^{\infty}\left[1+\frac{\varepsilon-e \Phi(\mathbf{r})}{\kappa_{0} k_{B} T_{e}+e \bar{\Phi}_{e}}\right]^{-\kappa_{0}-\frac{5}{2}} \Theta\left[\varepsilon-e \Phi(\mathbf{r})+e \bar{\Phi}_{e}+\kappa_{0} k_{B} T_{e}\right] \varepsilon^{\frac{1}{2}} d \varepsilon}{\int_{0}^{\infty}\left(1+\frac{\varepsilon}{\kappa_{0} k_{B} T_{e}+e \bar{\Phi}_{e}}\right)^{-\kappa_{0}-\frac{5}{2}} \Theta\left[\varepsilon-e \Phi(\mathbf{r})+e \bar{\Phi}_{e}+\kappa_{0} k_{B} T_{e}\right] \varepsilon^{\frac{1}{2}} d \varepsilon}, \\
n_{i}\left(\mathbf{r} ; \kappa_{0}, T_{i}\right)=n_{\infty} \cdot \frac{\int_{\varepsilon M i(\mathbf{r})}^{\infty}\left[1+\frac{\varepsilon+e \Phi(\mathbf{r})}{\kappa_{0} k_{B} T_{i}-e \bar{\Phi}_{e}}\right]^{-\kappa_{0}-\frac{5}{2}} \Theta\left[\varepsilon+e \Phi(\mathbf{r})-e \bar{\Phi}_{e}+\kappa_{0} k_{B} T_{i}\right] \varepsilon^{\frac{1}{2}} d \varepsilon}{\int_{\varepsilon M i(\mathbf{r})}^{\infty}\left(1+\frac{\varepsilon}{\kappa_{0} k_{B} T_{i}-e \bar{\Phi}_{e}}\right)^{-\kappa_{0}-\frac{5}{2}} \Theta\left[\varepsilon+e \Phi(\mathbf{r})-e \bar{\Phi}_{e}+\kappa_{0} k_{B} T_{i}\right] \varepsilon^{\frac{1}{2}} d \varepsilon} .
\end{gathered}
$$

Equations (15a) and (15b) describe the ion and electron densities and are consistently defined for any large values of the potential, in contrast to (8a) and (8b). 


\subsection{Weakly coupled plasmas}

The two previous issues, the small charge perturbations, $e \Phi(r) \ll \frac{\kappa_{0}}{\kappa_{0}+1} k_{\mathrm{B}} T_{i, e}$, and the cut-off of the electron and ion densities for $\kappa_{0} k_{B} T_{i, e} \pm e\left[\Phi(\mathbf{r})-\bar{\Phi}_{i, e}\right]<0$, are both equivalent to the requirement of large number of particles in the Debye volume, $N_{\mathrm{D}}$. In fact, both of these issues require sufficiently small perturbations in order to be resolved, i.e.

$$
\frac{e \Phi(\mathbf{r})}{\kappa_{0} k_{B} T_{e}}=\frac{t_{i}}{\kappa_{0}+1} \cdot \frac{\Delta N}{N_{\mathrm{D}}} \cdot \frac{\exp \left(-r / \lambda_{\mathrm{D}, \mathrm{K}}\right)}{r / \lambda_{\mathrm{D}, \mathrm{K}}} \leqslant \frac{\Delta N}{N_{\mathrm{D}}} \cdot \frac{\lambda_{\mathrm{D}, \mathrm{K}}}{b}=\frac{\Delta N}{N_{\mathrm{D}}^{\frac{2}{3}}}\left(\frac{4 \pi}{3}\right)^{\frac{1}{3}}<1,
$$

where we maximize $e \Phi(\mathbf{r}) /\left(\kappa_{0} k_{B} T_{e}\right)$ by considering the extreme cases of $\kappa_{0} \rightarrow 0$, $t_{i} \equiv T_{i} /\left(T_{i}+T_{e}\right) \rightarrow 1$, and $r \rightarrow b$ (interparticle distance). The inequality (16) is equivalent to the requirement of large $N_{D}$,

$$
N_{\text {D }}>\left(\frac{4 \pi}{3}\right)^{\frac{1}{2}} \Delta N^{\frac{3}{2}}
$$

The number of ions that contribute to the charge perturbation can vary from $\sim 1$ to orders of magnitude larger, and theoretically reach even the total number of particles in a Debye sphere, $N_{\mathrm{D}}$. However, a likely charge perturbation is $\frac{1}{2} \sqrt{N_{\mathrm{D}}}$, which comes from the fact that all the particles in a Debye sphere are correlated and behave like a cluster of particles within the overall system (Livadiotis and McComas 2011b, 2013a; see also Fahlen et al. 2011). Indeed, let us consider an imaginary separatrix that divides a Debye sphere into two halves, with all the particles located at one side at a given time $t=t_{0}$, and for each of the $N_{D}$ particles there is a $p=1 / 2$ probability to be on the one or the other side. At some time later, $t \gg t_{0}$, the equilibrium is obtained between the two sides, and the probability of $N$ particles located on one side is given by the binomial distribution, $P(N)=\left(2^{-N_{\mathrm{D}}} / N_{\mathrm{D}} !\right) \cdot N ! /\left(N_{\mathrm{D}}-N\right)$ !. The mean value $\langle N\rangle=\frac{1}{2} N_{\mathrm{D}}$, which is also the most frequent $N$ (for $N_{\mathrm{D}} \gg 1$ ), has standard deviation $\sqrt{\left\langle\Delta N^{2}\right\rangle}=\frac{1}{2} \sqrt{N_{\mathrm{D}}}$, and relative standard deviation $1 / \sqrt{N_{\mathrm{D}}}$ that tends to zero for $N_{D} \gg 1$.

Considering a charge perturbation of particles $\sim \frac{1}{2} \sqrt{N_{\mathrm{D}}}$, the inequality (17) becomes $N_{\mathrm{D}}>\left(\frac{\pi}{6}\right)^{2}$, which is consistent with the requirement for weakly coupled plasmas $N_{\mathrm{D}} \gg 1$, that is equivalent to $\lambda_{\mathrm{D} \infty} \gg b$. Given that $N_{\mathrm{D}}=N_{\mathrm{D} \infty}\left(\frac{\kappa_{0}}{\kappa_{0}+1}\right)^{\frac{3}{2}}$, with $N_{\mathrm{D} \infty} \equiv$ $N_{\mathrm{D}}\left(\kappa_{0} \rightarrow \infty\right)=\frac{4 \pi}{3} n_{\infty} \lambda_{\mathrm{D} \infty}^{3}$, the restriction $N_{\mathrm{D}} \gg 1$ becomes

$$
\kappa_{0} \gg N_{\mathrm{D} \infty}^{-\frac{2}{3}}
$$

e.g. for a plasma of $N_{\mathrm{D} \infty} \sim 10^{6}$, the kappa index must be $\kappa_{0} \gg 10^{-4}$. In fact, inequality (18a) is a restriction between the plasma thermodynamic parameters, which must be held for any weakly coupled plasma (Fig. 6),

$$
\kappa_{0} T n^{-\frac{1}{3}} \gg\left(\frac{4 \pi}{3}\right)^{-\frac{2}{3}} \frac{e^{2}}{\varepsilon k_{\mathrm{B}}} \cong 8.1 \times 10^{-3}
$$

(with temperature given in $\mathrm{K}$ and density in $\mathrm{cm}^{-3}$ ).

\subsection{No correlations between ions and electrons}

The specific formulation of densities, as used in (8a), implies that the electrons are not correlated with ions. This is because in all the derivations of the Debye length, the one-particle formulation of kappa distributions has been used, instead of the 


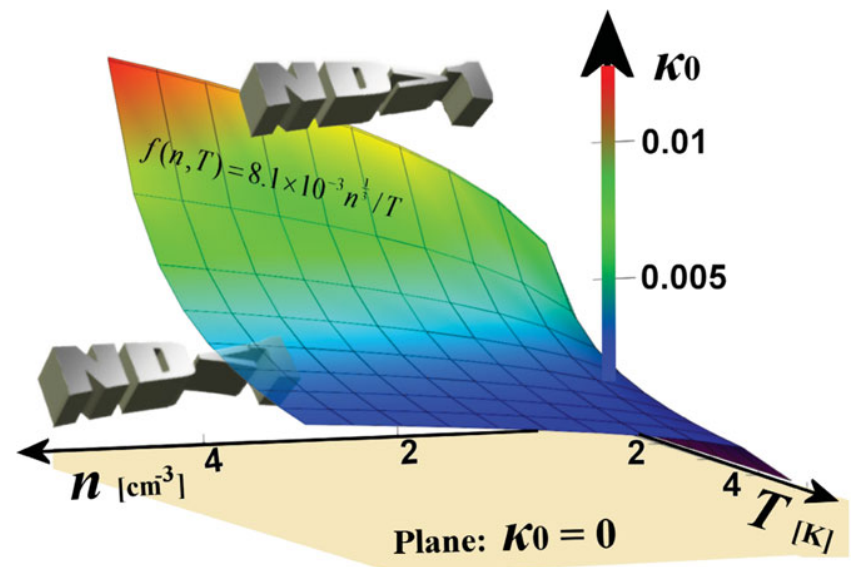

FiguRE 6. Plot of the surface $f(n, T)=8.1 \times 10^{-3} n^{\frac{1}{3}} / T$ that separates the kappa indices $\kappa_{0} \gg f(n, T)$ of a weakly coupled plasma $\left(N_{\mathrm{D}} \gg 1\right)$ from the kappa indices $\kappa_{0} \ll f(n, T)$ of a strongly coupled plasma $\left(N_{\mathrm{D}} \ll 1\right)$. The weakly coupled plasma is a system with a collective behavior between particles. (Note that the same surface indicates the kappa indices that correspond to small charge perturbations $N_{\mathrm{D}} \gg 1$.)

more complicated $N$-particle formulation (Livadiotis and McComas 2011b, 2013b). At least, a two-particle distribution must be used if we would like to subscribe a correlation between electrons and ions. Then, three types of correlation may be identified: (1) ion-ion, (2) electron-electron, and (3) ion-electron. Since, the kappa index characterizes the correlation between some particle species, we may also have three different kappa indices, i.e. $\kappa_{0}^{i}$ (ion-ion), $\kappa_{0}^{e}$ (electron-electron), and $\kappa_{0}^{i-e}$ (ionelectron). Then, the whole distribution is written as (appendix A in Livadiotis and McComas 2013b),

$$
P\left(\mathbf{r}_{i, e}, \mathbf{u}_{i, e} ; \kappa_{0}^{i, e}, T_{i, e} ; \kappa_{0}^{i-e}\right) \sim\left\{\sum_{S=i, e}\left[1+\frac{1}{\kappa_{0}^{S}} \cdot \frac{H\left(\mathbf{r}_{S}, \mathbf{u}_{S}\right)}{k_{B} T_{S}}\right]^{\frac{\kappa_{0}^{S}+2.5}{\kappa_{0}^{i-e}+2.5}}-1\right\}^{-\left(\kappa_{0}^{i-e}+2.5\right)} .
$$

So far, only the simple case of $\kappa_{0}^{i}=\kappa_{0}^{e} \equiv \kappa_{0}$ and $\kappa_{0}^{i-e} \rightarrow \infty$ has been studied.

\subsection{Existence of temperature for plasmas out of thermal equilibrium}

One of the main problems for defining the Debye length in non-equilibrium plasmas is the concept of temperature. Can a temperature be well-defined for systems out of thermal equilibrium? This issue has been thoroughly addressed for systems out of thermal equilibrium that reside in stationary states described by kappa distributions, or a superposition of those (Livadiotis and McComas 2009, 2010a).

The study of empirical kappa distributions and their connection with the modern framework of non-extensive Statistical Mechanics (Livadiotis and McComas 2009, 2012; appendix A in Livadiotis and McComas 2013b) has revealed that there is only one kappa distribution formulation (among many empirical ones) aligned with the principles of this statistical mechanics (e.g. escort probability, physical temperature). Namely,

$$
P\left(\mathbf{u} ; \mathbf{u}_{b} ; \theta, \kappa_{0}\right)=\left(\pi \kappa_{0} \theta^{2}\right)^{-\frac{3}{2}} \cdot \frac{\Gamma\left(\kappa_{0}+\frac{5}{2}\right)}{\Gamma\left(\kappa_{0}+1\right)} \cdot\left[1+\frac{1}{\kappa_{0}} \cdot \frac{\left(\mathbf{u}-\mathbf{u}_{b}\right)^{2}}{\theta^{2}}\right]^{-\kappa_{0}-\frac{5}{2}},
$$


where the thermal parameter $\theta$ has no dependence on the kappa index, and simply denotes the temperature in speed units, i.e. $\theta \equiv \sqrt{2 k_{\mathrm{B}} T / m}$; (20) coincides with the empirical form introduced by Vasyliũnas (1968).

This velocity distribution function, (20), is correctly connected with the framework of Tsallis non-extensive Statistical Mechanics (Tsallis 1988; Tsallis et al. 1998; Tsallis 2009), as shown by Livadiotis and McComas (2009). The statistical mechanics basis of kappa distributions is connected with the thermodynamics, and this connection is violated when the temperature is ill-defined. Beyond the kinetic definition of temperature, $\langle\varepsilon\rangle=\left\langle\frac{1}{2} m\left(\mathbf{u}-\mathbf{u}_{b}\right)^{2}\right\rangle=\frac{3}{2} k_{\mathrm{B}} T$, there is another definition of temperature, usually called thermodynamic definition because it connects the entropy $S$ with the internal energy $U\left(U=\langle\varepsilon\rangle\right.$, in the approximation of small potential), $T \equiv(\partial S / \partial U)^{-1}$. $\left[1-\frac{1}{\kappa} \cdot S / k_{\mathrm{B}}\right]$. This definition is consistent with the zeroth law of thermodynamics (Abe 2001). A well-defined temperature must be uniquely defined, and the fact of the equivalence of the two definition leads to a meaningful temperature for systems out of thermal equilibrium that are described by kappa distributions. (For more details on this topic, see Livadiotis and McComas 2009, 2010a, 2011b, 2012, 2013b; see also Livadiotis 2009; Livadiotis et al. 2011, 2012, 2013.)

\subsection{Isotropic Debye shielding}

In all the above, we have assumed that the Debye length is isotropic, namely, it is the same for any direction $\Omega \equiv(\vartheta, \varphi)$. The Debye length may vary with respect to the temperature, the density, and the kappa index. The temperature is a scalar field $T(r)$ that can often induce anisotropies.

The induced thermal anisotropy is sometimes interpreted as a 3-D temperature. However, the temperature cannot be defined as a vector or any other anisotropic mathematical element. The kinetic definition of temperature is given through the mean kinetic energy, $\frac{1}{2} m\left\langle u^{2}\right\rangle$, which is the mean of the sum (or the sum of the mean) of all the kinetic components, $\frac{1}{2} m\left\langle u_{x}^{2}+u_{y}^{2}+u_{z}^{2}\right\rangle$. Portions of kinetic energy may transfer from one component to the other, and thus, each component alone, $\frac{1}{2} m\left\langle u_{i}^{2}\right\rangle, i=x, y, z$, may not be an invariant quantity, even if the total kinetic energy remains invariant. However, the thermodynamic definition of temperature involves the mean of the total kinetic energy, i.e. over all the kinetic degrees of freedom. Even for systems where the classical theorem of the equidistribution of the (kinetic) energy in all the (kinetic) degrees of freedom is not valid, i.e. $\frac{1}{2} m\left\langle u_{i}^{2}\right\rangle$ depends on $i$, the kinetically defined temperature is still meaningful because it refers to the total kinetic energy, $\frac{3}{2} k_{\mathrm{B}} T \equiv \frac{1}{2} m\left\langle u_{x}^{2}+u_{y}^{2}+u_{z}^{2}\right\rangle$, or more precisely, to the average kinetic energy over all the degrees of freedom $f$, i.e.

$$
T \equiv \frac{1}{f} \sum_{i=1}^{f} T_{i}, \text { with } \frac{1}{2} k_{\mathrm{B}} T_{i} \equiv \frac{1}{2} m\left\langle u_{i}^{2}\right\rangle,
$$

or, in terms of speed units,

$$
\theta^{2} \equiv \frac{1}{f} \sum_{i=1}^{f} \theta_{i}^{2}, \text { with } \frac{1}{2} \theta_{i}^{2} \equiv\left\langle u_{i}^{2}\right\rangle .
$$

The anisotropic Debye length can be defined without the necessity of a vector-like temperature, such as the triad $\left(T_{x}, T_{y}, T_{z}\right)$. The temperature's scalar field, $T(x, y, z)$, can create anisotropies. For example, consider the 2-D polar space $(r, \vartheta) \in \mathfrak{R}^{2}$, and the 
specific case where the temperature scalar field depends only on the angular direction, $T(\vartheta)$. The Debye length can inherit this anisotropy, $\lambda_{\mathrm{D}}^{2}(\vartheta)=\left(k_{\mathrm{B}} \varepsilon e^{-2} n_{\infty}^{-1}\right) \cdot T(\vartheta)$.

In general, beyond the temperature anisotropy $T=T(\mathbf{r})$, the density and the medium can be also anisotropic, i.e. $n_{\infty}=n_{\infty}(\mathbf{r})$ and $\varepsilon(\mathbf{r})=\varepsilon_{r}(\mathbf{r}) \varepsilon_{0}$, where $\varepsilon_{r}$ is the relative permittivity. Hence, the anisotropic Debye length is now written as

$$
\lambda_{\mathrm{D} \infty}(\mathbf{r})=\lambda_{\mathrm{D} \infty}(\mathbf{r}) \cdot \sqrt{\frac{\kappa_{0}(\mathbf{r})}{\kappa_{0}(\mathbf{r})+1}}, \lambda_{\mathrm{D} \infty}(\mathbf{r}) \equiv \sqrt{\frac{k_{\mathrm{B}} \varepsilon_{0}}{e^{2}} \cdot \frac{\varepsilon_{r}(\mathbf{r}) T(\mathbf{r})}{n_{\infty}(\mathbf{r})}} .
$$

In the specific case of an angular dependent temperature scalar field, $T(\vartheta, \varphi)$, the Debye length is given by the 2-D Debye surface $\lambda_{\mathrm{D} \infty}(\vartheta, \varphi)=\sqrt{\left(k_{\mathrm{B}} \varepsilon e^{-2} n_{\infty}^{-1}\right) \cdot T(\vartheta, \varphi)}$,

$$
\lambda_{\mathrm{D}}(\vartheta, \varphi)=\lambda_{\mathrm{D} \infty}(\vartheta, \varphi) \cdot \sqrt{\frac{\kappa_{0}}{\kappa_{0}+1}}, \lambda_{\mathrm{D} \infty}(\vartheta, \varphi)=\sqrt{\left(k_{\mathrm{B}} \varepsilon e^{-2} n_{\infty}^{-1}\right) \cdot T(\vartheta, \varphi)},
$$

that generalizes the Debye sphere. An ellipsoid $(p=2)$, a superellipsoid $(p>2)$, or a subellipsoid $(p<2)$, are some simple cases of the generalized 2-D Debye surface,

$$
\frac{1}{\lambda_{\mathrm{D}}^{p}}=\left|\frac{\sin \vartheta \cos \varphi}{\alpha_{x}}\right|^{p}+\left|\frac{\sin \vartheta \sin \varphi}{\alpha_{y}}\right|^{p}+\left|\frac{\cos \vartheta}{\alpha_{z}}\right|^{p},
$$

where $\left(\alpha_{x}, \alpha_{y}, \alpha_{z}\right)$ are the three ellipsoid axes.

Frequently, space plasmas have particle populations described by anisotropic velocity distributions (for example, due to the presence of a local magnetic field). In these cases, the mean kinetic energy differs over the three kinetic degrees of freedom. Typically, one degree of freedom is a motion along the direction of the magnetic field $\left(u_{\mathrm{II}}\right)$, while the other two degrees span the perpendicular plane $\left(u_{\perp}\right)$. The thermal anisotropy of these plasmas is often interpreted by a vector-like temperature, $\left(T_{\mathrm{II}}, T_{\perp}\right)$ (e.g. Baumjohann and Treumann 2012). The actual temperature, however, must be given by $T=\left(T_{\mathrm{II}}+2 T_{\perp}\right) / 3$, where the perpendicular component is subject to two degrees of freedom that span the perpendicular plane. Moreover, the thermal anisotropy can be interpreted using the classical temperature scalar field, instead of the vector-like temperature. Indeed, by setting an angular scalar field, $T(\vartheta)$, where $\vartheta=0$ indicates the parallel direction and $\vartheta=\pi / 2$ the perpendicular plane, is sufficient to express mathematically the different temperature components $T_{\mathrm{II}}=T(\vartheta=0)$ and $T_{\perp}=T(\vartheta=\pi / 2)$; in addition, it can explain the temperature at any other directıon $\vartheta$.

The kappa distribution function for an anisotropic non-equilibrium plasma can be written using a vector-like temperature, as

$$
\begin{aligned}
& P\left(\mathbf{u} ; \mathbf{u}_{b}\right) \sim\left[1+\frac{1}{\kappa_{0, \mathrm{II}}} \cdot \frac{\frac{1}{2} m\left(u_{\mathrm{II}}-u_{b, \mathrm{II}}\right)^{2}}{k_{\mathrm{B}} T_{\mathrm{II}}}\right]^{-\kappa_{0, \mathrm{II}}-1.5} \\
& \quad \times\left[1+\frac{1}{\kappa_{0, \perp}} \cdot \frac{\frac{1}{2} m\left(\mathbf{u}_{\perp}-\mathbf{u}_{b, \perp}\right)^{2}}{k_{\mathrm{B}} T_{\perp}}\right]^{-\kappa_{0, \perp}-2} d u_{\mathrm{II}} u_{\perp} d u_{\perp} d \varphi,
\end{aligned}
$$

which treats the parallel and the perpendicular directions as uncorrelated. For simplicity, the two component kappa index may be taken to be the same, $\kappa_{0, \mathrm{II}}=\kappa_{0, \perp}=\kappa_{0}$. One specific case is for plasmas at thermal equilibrium, where (24a) is written as a product of Maxwellian distributions (e.g. Krall and Trivelpiece 
1973; Baumjohann and Treumann 2012),

$$
P\left(\mathbf{u} ; \mathbf{u}_{b}\right) \sim \exp \left[-\frac{\frac{1}{2} m\left(u_{\mathrm{II}}-u_{b, \mathrm{II}}\right)^{2}}{k_{\mathrm{B}} T_{\mathrm{II}}}-\frac{\frac{1}{2} m\left(\mathbf{u}_{\perp}-\mathbf{u}_{b, \perp}\right)^{2}}{k_{\mathrm{B}} T_{\perp}}\right] d u_{\mathrm{II}} u_{\perp} d u_{\perp} d \varphi .
$$

As explained above, (24a) and (24b) imply a vector-like temperature and kappa index, which is not correct. In contrast, the correct expression must use thermodynamic variables as scalars instead of vector fields, e.g. $T(\vartheta, \varphi)$ and $\kappa_{0}(\vartheta, \varphi)$,

$$
P\left(\mathbf{u} ; \mathbf{u}_{b}\right) \sim\left[1+\frac{1}{\kappa_{0}(\vartheta, \varphi)} \cdot \frac{\frac{1}{2} m\left(\mathbf{u}-\mathbf{u}_{b}\right)^{2}}{k_{\mathrm{B}} T(\vartheta, \varphi)}\right]^{-\kappa_{0}(\vartheta, \varphi)-2.5} u^{2} d u \sin \vartheta d \vartheta d \varphi .
$$

\section{Modifications of the two known interpretations}

\subsection{Inconsistencies and modifications of Interpretation 1}

Interpretation 1 involves the ratio of the potential to thermal energy, $R$, normalized by the perturbation charge $\Delta N$ that causes the potential $\Phi$, or more precisely, by the ratio $\Delta N / N_{\mathrm{D}}$ as shown in (2). In order to derive the Debye length via Interpretation 1 , it is necessary to solve the Poisson (10) and find the formulation of the potential $\Phi$. Then, the Debye length is the distance from the charge perturbation for which the potential energy $e \Phi$ becomes comparable with the thermal energy, and more specifically, the distance where the ratio of the potential to thermal energy, $R$, as defined in (2), is a constant of the order of unity, (5a). However, (12b) shows that $R\left(\lambda_{\mathrm{D}, \mathrm{K}}\right)$ is proportional to $\frac{\kappa_{0}}{\kappa_{0}+1}$, and thus it is not a constant and not of the order of unity (at least for small kappa indices, $\kappa_{0} \ll 1$ ). Therefore, Interpretation 1 leads to a well-defined Debye length for plasmas at thermal equilibrium $\left(\kappa_{0} \rightarrow \infty\right)$, or at least, for plasmas at near-equilibrium $\left(\kappa_{0}>1\right)$, for which the dependence of $R$ on the kappa index is less significant.

This inconsistency of Interpretation 1 can be solved by modifying the formulation of $R$ that is given in (2). First, we recall that the approximation of small charge perturbation requires the quantity $e \Phi /\left(k_{\mathrm{B}} T_{0}\right)$ to be small, for equilibrium plasmas, and this is modified to $e \Phi /\left(\frac{\kappa_{0}}{\kappa_{0}+1} k_{\mathrm{B}} T_{0}\right)$ for non-equilibrium plasmas. This quantity is small because the perturbation charge is considered small, i.e. $\Delta N / N_{\mathrm{D}} \ll 1$, and thus, their ratio is expected to be some constant. In fact, for equilibrium plasmas, their ratio $e \Phi /\left(k_{\mathrm{B}} T_{0} \frac{\Delta N}{N_{\mathrm{D}}}\right)$ coincides with the ratio $\frac{1}{2} R$, given in (2). Therefore, for non-equilibrium plasmas, a consistent modification of this ratio can be given from the quantity $e \Phi /\left(\frac{\kappa_{0}}{\kappa_{0}+1} k_{\mathrm{B}} T_{0}\right)$, normalized by $\Delta N / N_{\mathrm{D}}$, i.e.

$$
\tilde{R} \equiv \frac{e \Phi}{\frac{1}{2} k_{\mathrm{B}} T_{0}} \cdot \frac{1}{\frac{\kappa_{0}}{\kappa_{0}+1}} \cdot \frac{1}{\frac{\Delta N}{N_{\mathrm{D}}}},
$$

(where $\left.\tilde{R} \equiv R\right|_{\text {modified }}$ ); then, at the Debye length, this ratio is a constant of the order of unity,

$$
\tilde{R}\left(\lambda_{\mathrm{D}, \mathrm{K}}\right) \cong \text { constant }=C(d) \cdot(1 / \mathrm{e}) .
$$

\subsection{Inconsistencies and modifications of Interpretation 2}

Interpretation 2 is based on the arbitrary threshold (1/e) that equals the ratio of the shielded over the unshielded potential, $\Phi\left(\lambda_{\mathrm{D}, \Lambda}\right) / \Phi_{\mathrm{us}}=1 / \mathrm{e}$, at the Debye length, (5b). One way to avoid including a threshold can be realized by using the differential 


\begin{tabular}{|c|c|c|c|}
\hline Interpretation & Inconsistency & Modification & Result \\
\hline \multirow[t]{2}{*}{1} & $\begin{array}{l}\text { The ratio of potential } \\
\text { to thermal energy, } R \text {, } \\
\text { depends on the } \\
\text { kappa index }\end{array}$ & $\tilde{R} \equiv \frac{e \Phi}{\frac{1}{2} k_{\mathrm{B}} T_{0}} \cdot \frac{1}{\frac{\kappa_{0}}{\kappa_{0}+1}} \cdot \frac{1}{\frac{\Delta N}{N_{\mathrm{D}}}}$ & $\begin{array}{l}\text { The Debye length is at } \\
\text { that distance from the } \\
\text { perturbation for which } \\
\text { the ratio } \tilde{R} \text { is given by } \\
\text { some constant of the } \\
\text { order of unity, } \\
\sim C(d) \cdot(1 / \mathrm{e}) .\end{array}$ \\
\hline & $\begin{array}{l}\text { The shielding is held } \\
\text { for small } \\
\text { perturbations }\end{array}$ & $\tilde{R} \equiv \lim _{\Phi \rightarrow 0}\left(\frac{e \Phi}{\frac{1}{2} k_{\mathrm{B}} T_{0}} \cdot \frac{1}{\frac{\kappa_{0}}{\kappa_{0}+1}} \cdot \frac{1}{\frac{\Delta N}{N_{\mathrm{D}}}}\right)$ & \\
\hline \multirow[t]{2}{*}{2} & $\begin{array}{l}\text { The ratio of the } \\
\text { shielded to the } \\
\text { unshielded potential } \\
\text { equals an arbitrary } \\
\text { threshold }\end{array}$ & & $\begin{array}{l}\text { The Debye length is } \\
\text { given by the ratio of } \\
\text { the potential } \Phi \\
\text { (outcome of shielding) } \\
\text { over the charge } \\
\text { distribution due to } \\
\text { excess of electrons } \\
e\left(n_{e}-n_{i}\right) \text { (cause of } \\
\text { shielding) }\end{array}$ \\
\hline & $\begin{array}{l}\text { The shielding is held } \\
\text { for small } \\
\text { perturbations }\end{array}$ & $\lim _{\Phi \rightarrow 0} \frac{\Phi}{\frac{1}{\varepsilon} \cdot e\left(n_{e}-n_{i}\right)}=\lambda_{\mathrm{D}, \Lambda}^{2}$ & \\
\hline
\end{tabular}

TABLE 4. Inconsistencies and modifications of the two interpretations of the Debye length.

equation rather than its solution, i.e.

$$
\lim _{\Phi \rightarrow 0} \frac{\Phi}{\left|\nabla^{2} \Phi\right|}=\lambda_{\mathrm{D}, \Lambda}^{2} .
$$

Here, the threshold 1/e that determines the Debye length is 'encrypted' in the differential equation (26) and the exponential form of its solution. However, we could substitute $\nabla^{2} \Phi$ with its equivalent form from the Poisson (10). Therefore, a more general way to express Interpretation 2 is via the ratio of the 'outcome', the potential $\Phi$, to its 'cause', the non-zero charge distribution $e\left(n_{e}-n_{i}\right)$, i.e.

$$
\lim _{\Phi \rightarrow 0} \frac{\Phi}{\frac{1}{\varepsilon} \cdot e\left(n_{e}-n_{i}\right)}=\lambda_{\mathrm{D}, \Lambda}^{2} .
$$

Smaller perturbation charge $\Delta N$ leads to weaker potential $\Phi$ and to weaker charge distribution $e\left(n_{e}-n_{i}\right)$. Consequently, these two quantities converge to being exactly proportional as $\Delta N \rightarrow 0$ (or $\Phi \rightarrow 0$ ). The proportionality coefficient does not depend on either of them, but instead is characterized by thermodynamic parameters of the non-perturbed plasma (density, temperature, kappa); this provides a more robust interpretation for the Debye length (its square), as shown in (27).

\subsection{Synopsis of inconsistencies and modifications}

Table 4 gathers the inconsistencies and modifications of the two interpretations explained above.

We have modified the two interpretations to be consistent for both equilibrium and non-equilibrium plasmas, and for any dimensionality of the charge perturbation. 
However, they are still characterized by certain restrictions. For example, Interpretation 1 needs the formulation of the potential. Indeed, the Poisson equation has to be solved and find the potential energy first, before this is compared to the thermal energy. Also, Interpretation 1 is restricted for non-equilibrium plasmas that are described by a single kappa distribution. On the other hand, Interpretation 2 is not restricted to any specific forms of the ion/electron distribution functions. The following application of a superposition of kappa distributions illuminates this flexibility of Interpretation 2.

\subsection{Application: superposition of kappa distributions}

The densities given in terms of a single kappa distribution, (8b), can be generalized to a linear superposition on kappa distributions with density of kappa indices $D_{i, e}\left(\kappa_{0}\right)$, i.e.

$$
\begin{aligned}
& n_{i}=n_{\infty} \cdot \sum_{\kappa_{0}} D_{i}\left(\kappa_{0}\right) \cdot\left[1+\frac{e \Phi(\mathbf{r})}{\kappa_{0} k_{\mathrm{B}} T_{i}-e \bar{\Phi}_{i}}\right]^{-\kappa_{0}-1}, \\
& n_{e}=n_{\infty} \cdot \sum_{\kappa_{0}} D_{e}\left(\kappa_{0}\right) \cdot\left[1-\frac{e \Phi(\mathbf{r})}{\kappa_{0} k_{\mathrm{B}} T_{e}+e \bar{\Phi}_{e}}\right]^{-\kappa_{0}-1} .
\end{aligned}
$$

Given the approximation of small perturbations, the charge distribution is

$$
\rho=e\left(n_{e}-n_{i}\right)=e n_{\infty} \cdot \frac{e \Phi(r)}{k_{\mathrm{B}} T_{0}} \cdot\left\langle\frac{\kappa_{0}+1}{\kappa_{0}}\right\rangle,
$$

where

$$
\begin{gathered}
\left\langle\frac{\kappa_{0}+1}{\kappa_{0}}\right\rangle=\sum_{\kappa_{0}} D\left(\kappa_{0}\right) \cdot \frac{\kappa_{0}+1}{\kappa_{0}}, \text { and, } \\
D\left(\kappa_{0}\right) \equiv t_{e} \cdot D_{i}\left(\kappa_{0}\right)+t_{i} \cdot D_{e}\left(\kappa_{0}\right), t_{i, e} \equiv T_{i, e} /\left(T_{i}+T_{e}\right) .
\end{gathered}
$$

Then, utilizing Interpretation 2, we find that the generalized Debye length is given by

$$
\lambda_{\mathrm{D}, \Lambda}=\left\langle\frac{\kappa_{0}+1}{\kappa_{0}}\right\rangle^{-\frac{1}{2}} \cdot \lambda_{\mathrm{D} \infty},
$$

and the relevant potential (for any dimensionality $d$ ) is given by

$$
\frac{e \Phi(r)}{k_{\mathrm{B}} T_{0}}=\left[d \Gamma\left(\frac{d+1}{2}\right)\right]^{-1} \cdot \frac{\Delta N}{N_{\mathrm{D}}} \cdot\left\langle\frac{\kappa_{0}+1}{\kappa_{0}}\right\rangle^{-1} \cdot \frac{\exp \left(-r / \lambda_{\mathrm{D}, \Lambda}\right)}{\left(r / \lambda_{\mathrm{D}, \Lambda}\right)^{(d-1) / 2}} .
$$

As an example, we consider the gamma distribution of kappa indices,

$$
D\left(\kappa_{0}\right)=\frac{1}{\Gamma(\mu+1)} \kappa_{0}^{\mu} e^{-\kappa_{0}},
$$

where $\mu$ is the most frequent kappa index. Then, we find

$$
\left\langle\frac{\kappa_{0}+1}{\kappa_{0}}\right\rangle^{-1}=\frac{\mu}{\mu+1}, \text { or, } \lambda_{\mathrm{D}, \Lambda}=\sqrt{\frac{\mu}{\mu+1}} \cdot \lambda_{\mathrm{D} \infty} .
$$

Hence, for a superposition of kappa indices with density given by (32), the usually modified Debye length $\lambda_{\mathrm{D}, \mathrm{K}}=\sqrt{\frac{\kappa_{0}}{\kappa_{0}+1}} \cdot \lambda_{\mathrm{D} \infty},(6)$, is replaced by $\lambda_{\mathrm{D}, \Lambda}=\sqrt{\frac{\mu}{\mu+1}} \cdot \lambda_{\mathrm{D} \infty}$, (33), namely, the kappa index $\kappa_{0}$ is simply replaced by its most frequent value $\mu$. 


\section{The third interpretation of the Debye length}

We have seen that Interpretation 2 is not restricted to any specific forms of the ion/electron distribution functions. The only requirement is that the specific forms of the distributions have to be known (either analytically or numerically). Here, we present a third interpretation of Debye length, for which only the second moment of the ion/electron distribution functions is necessary.

Livadiotis and McComas (2013a, 2013e) recently began developing the concept of organization of plasmas by their Debye shielding within clusters of locally correlated particles (Debye spheres). According to this, the Debye length assigns a type of large-scale uncertainty in position, $\delta x=\sqrt{\left\langle\Delta x^{2}>\right.} \sim \lambda_{\mathrm{D}}$, that leads to a large-scale uncertainty in time, $\delta t \sim \lambda_{\mathrm{D}} / \theta$. Given this time uncertainty and the least energy of a particle in a Debye sphere, these authors found a large-scale quantization of phase-space, some 12 orders of magnitude larger than the Planck constant.

In Appendix $\mathrm{F}$ we derive the average of any positional function $f(\mathbf{r})$, using the charge distribution in plasma $\rho(\mathbf{r})=e\left[n_{e}(\mathbf{r})-n_{i}(\mathbf{r})\right]$, with normalization given by the number of the excess electrons $\Delta N=\frac{1}{e} \int_{r \in V} \rho(\mathbf{r}) d V$ ( $d V$ is the elementary volume within the charge distribution). Then, we show that the standard deviation of the position in the charge distribution of the plasma is given by $\sqrt{<\Delta x^{2}>} \cong \lambda_{\mathrm{D}}$, as was arrived at by different means by Livadiotis and McComas (2013a). This result is for 1-D shielding $(d=1)$, while for any $d$, the positional variance and standard deviation becomes

$$
\left\langle\Delta r^{2}\right\rangle=\frac{d+1}{2} \cdot \lambda_{\mathrm{D}}^{2}, \sqrt{\left\langle\Delta r^{2}\right\rangle}=\sqrt{\frac{d+1}{2}} \cdot \lambda_{\mathrm{D}} .
$$

The approximation of small charge perturbation has been used, i.e. (34a) is written as

$$
\lim _{\Phi \rightarrow 0}\left\langle\Delta r^{2}\right\rangle=\frac{d+1}{2} \cdot \lambda_{\mathrm{D}}^{2}, \lim _{\Phi \rightarrow 0} \sqrt{\left\langle\Delta r^{2}\right\rangle}=\sqrt{\frac{d+1}{2}} \cdot \lambda_{\mathrm{D}} .
$$

The similarity of this equation with the kinetic definition of temperature (Livadiotis and McComas 2009, 2010a, 2013b) is remarkable,

$$
\lim _{\Phi \rightarrow 0}\left\langle\Delta r^{2}\right\rangle=\frac{f_{\mathrm{D}}}{2} \cdot \lambda_{\mathrm{D}}^{2} \Leftrightarrow \lim _{\Phi \rightarrow 0}\left\langle\Delta u^{2}\right\rangle=\frac{f_{\mathrm{K}}}{2} \cdot \theta^{2},
$$

where the degrees of freedom $f_{\mathrm{K}}$ in the case of temperature represent the per-particle kinetic degrees of freedom $f_{\mathrm{K}}=d$. The degrees of freedom $f_{\mathrm{D}}$ in the case of shielding, similarly, must represent the per-particle degrees of freedom that are necessary for the shielding; we interpret $f_{\mathrm{D}}=d+1$ as the $d$ positional degrees of freedom of the shielded particle (charge perturbation) and one degree of freedom for any given shielding particle, as its shielding always occurs on a line directly toward or away from the perturbation.

The third interpretation of the Debye length emerges naturally in a way analogous to the kinetic definition of temperature, as shown in (35). The advantage of this interpretation is that it can be used to derive the Debye length without having to know the whole ion/electron positional distributions but just their second statistical moment. This is similar to the concept of temperature, which can be derived from the second statistical moment of the velocity distributions without having to know the whole distribution.

Finally, the three interpretations of the Debye length are compared in Table 5 and illustrated schematically in Fig. 7. The Debye length can be derived from its three interpretations - definitions given in this paper. There are, however, several 


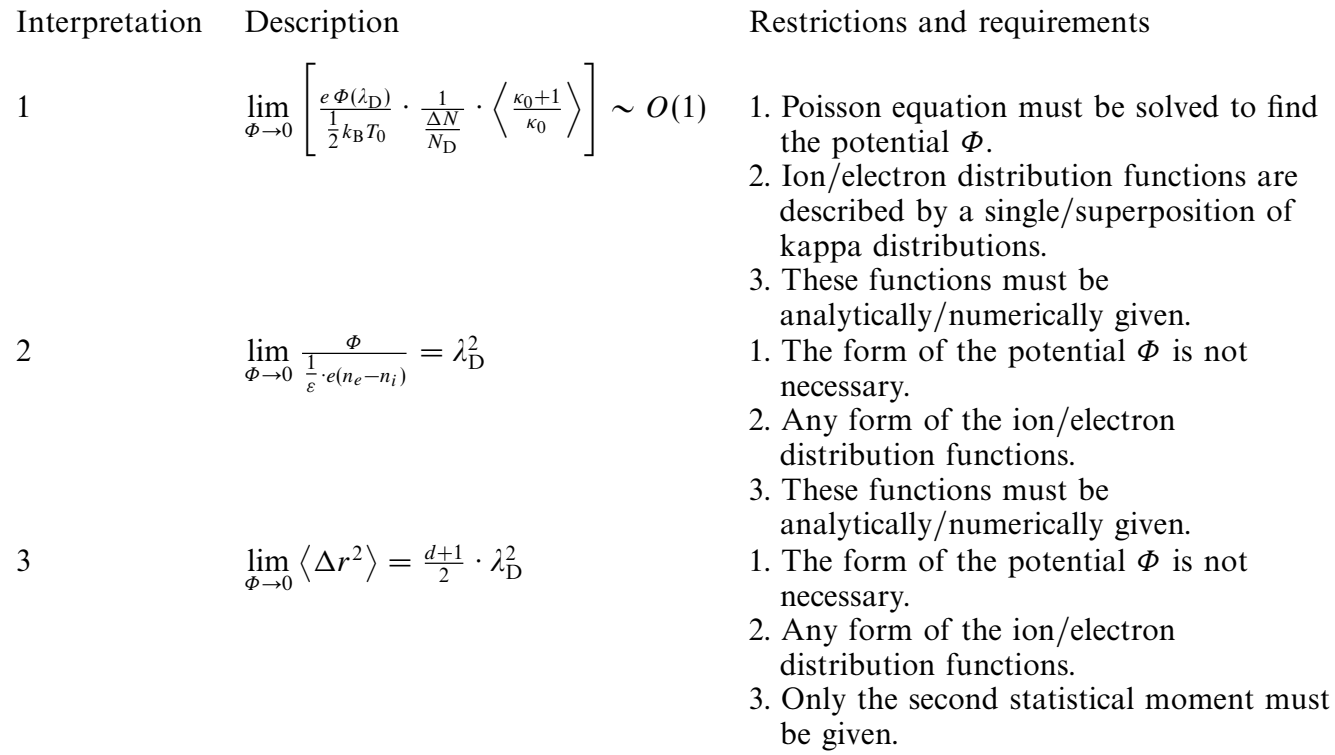

TABLE 5. The three interpretations of the Debye length.

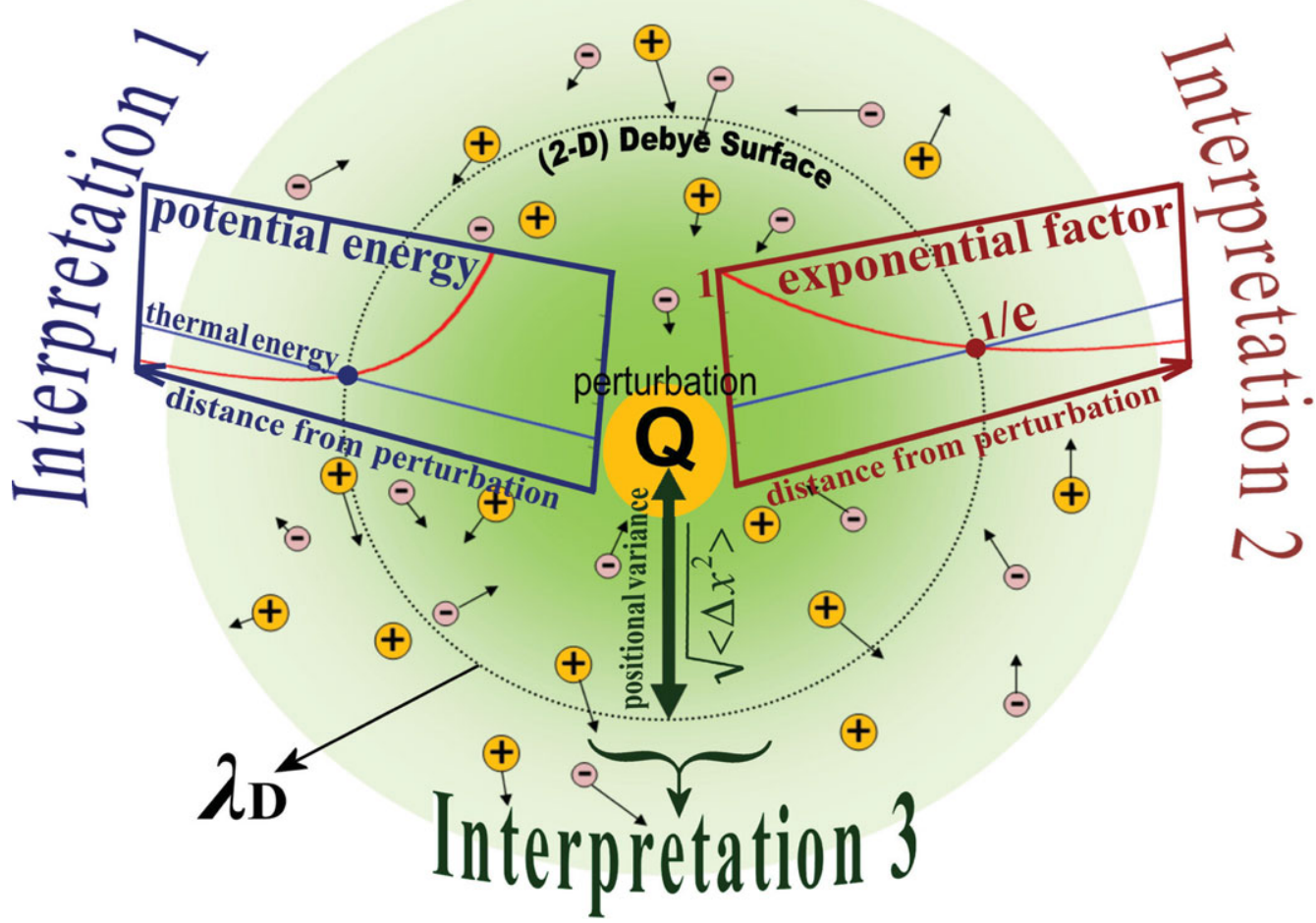

FIGURE 7. Schematic diagram of the three interpretations of the Debye length.

other interpretations of the Debye length, which are associated with its properties rather than providing new derivation methods. Namely, the Debye sphere may be interpreted as the region where (1) thermal fluctuations in plasma waves dominate 
any wave propagation; (2) plasma wave dispersion cannot be defined anymore; (3) electron orbits are undefined; and (4) it must by far exceed the interparticle space for a plasma to be defined properly $\left(N_{D} \gg 1\right)$.

\section{Conclusions}

In this paper we examined the interpretations of the Debye length for plasmas at, or out of thermal equilibrium, originating from the Poisson equation of Gauss' law for electrodynamics and its solution of the electric potential that is caused by small charge perturbations in the plasma. Two different interpretations can be found in the literature: (1) The ratio of the potential to the thermal energy is a constant of the order of unity, and (2) the ratio of the shielded to the unshielded potential is a constant of the order of unity (e.g. 1/e). The two interpretations of the Debye length have been examined for plasmas at thermal equilibrium, described by the BoltzmannMaxwell phase space distribution, and plasmas out of thermal equilibrium, described by the kappa distribution. We studied three different dimensionalities of the electric potential/field and the charge perturbation: (1) 1-D field $\mathbf{E}$ of linear symmetry for planar charge density, (2) 2-D field $\mathbf{E}$ of cylindrical symmetry for linear charge density, and (3) 3-D field $\mathbf{E}$ of spherical symmetry for a point charge perturbation. For each dimensionality, we derived the electric potential/field for both equilibrium and nonequilibrium plasmas, and then used this potential to examine the two interpretations.

In this study we showed the main resolutions and modifications of the two interpretations as follows:

- (i) The two interpretations are consistent with each other for both equilibrium and non-equilibrium plasmas and for all three dimensionalities, if the Debye length is characterized by the known modification that incorporates the kappa index, i.e. $\lambda_{\mathrm{D}, \mathrm{K}} \equiv \lambda_{\mathrm{D} \infty} \cdot \sqrt{\frac{\kappa_{0}}{\kappa_{0}+1}}$.

- (ii) Interpretation 1 is modified so that the ratio of the potential to thermal energy is normalized to be independent of the kappa index for plasmas out of thermal equilibrium.

- (iii) Both interpretations must be restricted to small charge perturbations for the ion/electron densities to be well-defined, which is equivalent to the condition of having weakly coupled plasmas.

- (iv) Non-equilibrium plasmas are described by a single kappa distribution. The more general case of linear superposition of kappa distributions was solved by replacing the single kappa index with averages of the kappa indices.

- (v) The ion/electron densities for non-equilibrium plasmas were expressed considering ion-ion and electron-electron but no ion-electron correlations. We showed how a general density function can be constructed that can include all three types of correlations.

- (vi) Debye shielding was previously considered to be isotropic. We developed the concept of an anisotropic Debye shielding and Debye length.

Using the concept of large-scale quantization, we showed that a third interpretation of the Debye length naturally emerges from the second statistical moment of the plasma charge density, that is, the positional distribution. This is similar to the kinetic definition of temperature, which is the second statistical moment of the velocity distribution. The advantage of this interpretation is that it can be used to derive the Debye length without having to know the whole ion/electron positional distributions but just their second statistical moment. 
Finally, we compare the three interpretations of the Debye length: Interpretation 1 needs the Poisson equation to be solved to derive the formulation of the potential, and applies for non-equilibrium plasmas described by single, or a superposition of, kappa distributions. Interpretation 2 is not restricted to any specific forms of the ion/electron distribution functions, but these forms have to be given, either analytically or numerically. Interpretation 3 needs only the second statistical moment.

Thus, by examining electrostatic shielding in plasmas in detail, we have resolved the fundamental physical meaning of the Debye length and defined what information is required for theoretical and experimental plasma-physics researchers to appropriately use each of the three interpretations of this key scale length.

\section{Acknowledgements}

This work was funded in part by the Advanced Composition Explorer (ACE) and the Interstellar Boundary explorer (IBEX) missions, which are parts of NASA's Explorer Program.

\section{Appendix A. Kappa distribution ion and electron densities}

The kappa distributions are based on the solid statistical background of nonextensive statistical mechanics (Livadiotis and McComas 2009, 2013b). In the Statistical Physics community, the kappa distribution is better known as $q$-exponential, $q$-Gaussian, or $q$-Maxwellian distribution (Tsallis 2009). These distributions describe systems that are in stationary states but out of thermal equilibrium. The one-particle kappa distribution, defined in the 2-D phase space spanned by $(\mathbf{r}, \mathbf{u})$, is given by (Livadiotis and McComas 2013b)

$$
P\left(\mathbf{r}, \mathbf{u} ; \mathbf{u}_{b} ; \kappa, T\right)=\mathrm{A}_{\mathrm{H}}\left(\kappa_{0}, T\right) \cdot\left[1+\frac{1}{\kappa_{0}} \cdot \frac{H\left(\mathbf{r}, \mathbf{u} ; \mathbf{u}_{b}\right)}{k_{B} T}\right]^{-\kappa_{0}-1-\frac{d}{2}},
$$

where the normalization constant is

$$
\mathrm{A}_{\mathrm{H}}\left(\kappa_{0}, T\right) \equiv\left(\pi \kappa_{0} k_{\mathrm{B}} T\right)^{-\frac{d}{2}} \cdot \frac{\Gamma\left(\kappa_{0}+1+\frac{d}{2}\right)}{\Gamma\left(\kappa_{0}+1\right)} \cdot \mathrm{A}_{\Phi}\left(\kappa_{0}, T\right),
$$

with the potential normalization constant being equal to

$$
\mathrm{A}_{\Phi}\left(\kappa_{0}, T\right) \equiv\left\{\int_{\mathbf{r} \in V}\left[1+\frac{1}{\kappa_{0}} \cdot \frac{\Phi(\mathbf{r})}{k_{\mathrm{B}} T}\right]^{-\kappa_{0}-1} d V\right\}^{-1},
$$

where $\kappa_{0}$ is the invariant kappa index, $d V$ is the elementary volume, and the position vector spans the whole volume $V$. In the above description, the potential energy is appropriately constructed so that $\bar{\Phi}=0$, otherwise, $\Phi(\mathbf{r})$ must be replaced by $\Phi(\mathbf{r})-\bar{\Phi}$, where $\bar{\Phi}$ is the average potential energy,

$$
\bar{\Phi}\left(\kappa_{0}, T\right) \equiv \mathrm{A}_{\Phi}\left(\kappa_{0}, T\right) \cdot \int_{\mathbf{r} \in V}\left[1+\frac{1}{\kappa_{0}} \cdot \frac{\Phi(\mathbf{r})}{k_{\mathrm{B}} T}\right]^{-\kappa_{0}-1} \cdot \Phi(\mathbf{r}) d V .
$$


When all the $d$ kinetic degrees of freedom are integrated, the remaining distribution of $\mathbf{r}$ describes only the $d$ degrees of freedom of the regular space,

$$
P(\mathbf{r} ; \kappa, T)=\mathrm{A}_{\Phi}\left(\kappa_{0}, T\right) \cdot\left[1+\frac{1}{\kappa_{0}} \cdot \frac{\Phi(\mathbf{r})-\bar{\Phi}\left(\kappa_{0}, T\right)}{k_{\mathrm{B}} T}\right]^{-\kappa_{0}-1} .
$$

Multiplying the regular space normalized distribution (A3) with the total number of particles $N$, we derive the particle density, $n=n\left(\mathbf{r} ; \kappa_{0}, T\right)$, given by

$$
n(\mathbf{r} ; \kappa, T)=N \cdot P(\mathbf{r} ; \kappa, T)=N \cdot \mathrm{A}_{\Phi}\left(\kappa_{0}, T\right) \cdot\left[1+\frac{1}{\kappa_{0}} \cdot \frac{\Phi(\mathbf{r})-\bar{\Phi}\left(\kappa_{0}, T\right)}{k_{\mathrm{B}} T}\right]^{-\kappa_{0}-1}
$$

Equation (A4) can be written in the simplified form

$$
n=\bar{n} \cdot\left(1+\frac{1}{\kappa_{0}} \cdot \frac{\Phi_{\mathrm{c}}}{k_{\mathrm{B}} T}\right)^{-\kappa_{0}-1}
$$

where $\Phi_{\mathrm{C}}\left(\mathbf{r} ; \kappa_{0}, T\right) \equiv \Phi(\mathbf{r})-\bar{\Phi}\left(\kappa_{0}, T\right)$ denotes the 'centric' potential, and $\bar{n} \equiv N \cdot \mathrm{A}_{\Phi}$ is the density for zero centric potential.

In quasi-neutral plasmas, we write (A5) separately for ions and electrons,

$$
\begin{aligned}
& n_{i}(\mathbf{r})=\bar{n}_{i} \cdot\left\{1+\frac{e}{\kappa_{0} k_{\mathrm{B}} T_{i}} \cdot\left[\Phi(\mathbf{r})-\bar{\Phi}_{i}\right]\right\}^{-\kappa_{0}-1}, \\
& n_{e}(\mathbf{r})=\bar{n}_{e} \cdot\left\{1-\frac{e}{\kappa_{0} k_{\mathrm{B}} T_{e}} \cdot\left[\Phi(\mathbf{r})-\bar{\Phi}_{e}\right]\right\}^{-\kappa_{0}-1},
\end{aligned}
$$

with $N=\int_{\mathbf{r} \in V} n_{i}(\mathbf{r}) d V=\int_{\mathbf{r} \in V} n_{e}(\mathbf{r}) d V$ and

$$
\begin{aligned}
& \bar{n}_{i}=N \cdot\left\{\int_{\mathbf{r} \in V}\left\{1+\frac{e}{\kappa_{0} k_{\mathrm{B}} T_{i}} \cdot\left[\Phi(\mathbf{r})-\bar{\Phi}_{i}\right]\right\}^{-\kappa_{0}-1} d V\right\}^{-1}, \\
& \bar{n}_{e}=N \cdot\left\{\int_{\mathbf{r} \in V}\left\{1-\frac{e}{\kappa_{0} k_{\mathrm{B}} T_{e}} \cdot\left[\Phi(\mathbf{r})-\bar{\Phi}_{e}\right]\right\}^{-\kappa_{0}-1} d V\right\}^{-1},
\end{aligned}
$$

where $\kappa_{0}$ is the invariant kappa index (Livadiotis and McComas 2011b), common for ions and electrons; ions are taken to be singly charged for simplicity. The average potential $\bar{\Phi}_{i}, \bar{\Phi}_{e}$ are given by

$$
\bar{\Phi}_{i}=\frac{1}{N} \cdot \int_{\mathbf{r} \in V} n_{i}(\mathbf{r}) \Phi(\mathbf{r}) d V, \bar{\Phi}_{e}=\frac{1}{N} \cdot \int_{\mathbf{r} \in V} n_{e}(\boldsymbol{r}) \Phi(\boldsymbol{r}) d V .
$$

Quasi-neutrality requires $n_{i} \cong n_{e}$ for some sufficiently long distance $r \gg b$ (with $b$ indicating the interparticle distance), which becomes exact, $n_{i}=n_{e}$, for the theoretical limit of $r \rightarrow \infty$. At this distance for which $n_{i}=n_{e}$, the potential is noted by $\Phi_{\infty}$. Then, using (A6), the equality $n_{i}=n_{e}$ gives

$$
\bar{n}_{i} \cdot\left[1+\frac{e}{\kappa_{0} k_{\mathrm{B}} T_{i}} \cdot\left(\Phi_{\infty}-\bar{\Phi}_{i}\right)\right]^{-\kappa_{0}-1}=\bar{n}_{e} \cdot\left[1-\frac{e}{\kappa_{0} k_{\mathrm{B}} T_{e}} \cdot\left(\Phi_{\infty}-\bar{\Phi}_{e}\right)\right]^{-\kappa_{0}-1},
$$


from where we find

$$
\Phi_{\infty}=\frac{\kappa_{0}}{e} \cdot \frac{\beta_{e} T_{e}-\beta_{i} T_{i}}{\beta_{i}+\beta_{e}}+\frac{\beta_{i} \bar{\Phi}_{i}+\beta_{e} \bar{\Phi}_{e}}{\beta_{i}+\beta_{e}}, \text { with } \beta_{i, e} \equiv \bar{n}_{i, e}^{1 /\left(\kappa_{0}+1\right)} /\left(k_{\mathrm{B}} T_{i, e}\right) .
$$

(Note that we have $\Phi_{\infty} \cong \bar{\Phi}$, if $\bar{\Phi}_{i} \cong \bar{\Phi}_{e} \equiv \bar{\Phi}$ and $\bar{n}_{i} \cong \bar{n}_{e}$.) Hence, the density $n_{\infty}$ at infinity $(r \rightarrow \infty)$, that is at sufficient large distance so that quasi-neutrality applies, is given by

$$
n_{\infty}=\bar{n}_{i} \cdot\left[1+\frac{e}{\kappa_{0} k_{\mathrm{B}} T_{i}} \cdot\left(\Phi_{\infty}-\bar{\Phi}_{i}\right)\right]^{-\kappa_{0}-1}=\bar{n}_{e} \cdot\left[1-\frac{e}{\kappa_{0} k_{\mathrm{B}} T_{e}} \cdot\left(\Phi_{\infty}-\bar{\Phi}_{e}\right)\right]^{-\kappa_{0}-1},
$$

and the ion and electron densities in (A6) can be written as

$$
\begin{aligned}
& n_{i}(\mathbf{r})=n_{\infty} \cdot\left\{1+\frac{e \cdot\left[\Phi(\mathbf{r})-\Phi_{\infty}\right]}{\kappa_{0} k_{\mathrm{B}} T_{i}-e\left(\bar{\Phi}_{i}-\Phi_{\infty}\right)}\right\}^{-\kappa_{0}-1}, \\
& n_{e}(\mathbf{r})=n_{\infty} \cdot\left\{1-\frac{e \cdot\left[\Phi(\mathbf{r})-\Phi_{\infty}\right]}{\kappa_{0} k_{\mathrm{B}} T_{e}+e\left(\bar{\Phi}_{e}-\Phi_{\infty}\right)}\right\}^{-\kappa_{0}-1} .
\end{aligned}
$$

The total charge density is $\rho=e\left(n_{i}-n_{e}\right)$ that can be expanded in terms of the ratio of the potential to the thermal energy, and keeping terms up to the first order, we have

$$
\begin{aligned}
\rho / \varepsilon & \cong-\frac{e^{2} n_{\infty}}{k_{\mathrm{B}} \varepsilon}\left(\frac{1}{T_{i}}+\frac{1}{T_{e}}\right) \cdot \frac{\kappa_{0}+1}{\kappa_{0}} \cdot\left(\Phi-\Phi_{\infty}\right) \\
& =-\frac{1}{\lambda_{\mathrm{D} \infty}^{2}} \cdot \frac{\kappa_{0}+1}{\kappa_{0}} \cdot\left(\Phi-\Phi_{\infty}\right)=-\frac{1}{\lambda_{\mathrm{D}, \mathrm{K}}^{2}} \cdot\left(\Phi-\Phi_{\infty}\right) .
\end{aligned}
$$

\section{Appendix B. Debye length in equilibrium plasmas}

For plasmas at thermal equilibrium, the ion/electron densities are given by the Boltzmann distribution of energy,

$$
n_{i}=n_{\infty} \cdot \exp \left\{-\frac{e\left[\Phi(\mathbf{r})-\Phi_{\infty}\right]}{k_{\mathrm{B}} T_{i}}\right\}, n_{e}=n_{\infty} \cdot \exp \left\{\frac{e\left[\Phi(\mathbf{r})-\Phi_{\infty}\right]}{k_{\mathrm{B}} T_{e}}\right\},
$$

where $n_{\infty}$ denotes again the ion or electron density and $\Phi_{\infty}$ the electric potential at sufficient large distance so that quasi-neutrality is valid. $\Phi_{\infty}$ can be considered as the potential at infinity, so that $\Phi_{\infty} \cong 0$, i.e.

$$
n_{i}=n_{\infty} \cdot \exp \left[-\frac{e \Phi(\mathbf{r})}{k_{\mathrm{B}} T_{i}}\right], n_{e}=n_{\infty} \cdot \exp \left[\frac{e \Phi(\mathbf{r})}{k_{\mathrm{B}} T_{e}}\right] .
$$

The total charge density is $\rho=e\left(n_{i}-n_{e}\right)$; this is expanded in terms of the ratio of the potential to the thermal energy, assuming that $e \Phi(\mathbf{r}) /\left(k_{\mathrm{B}} T_{0}\right) \ll 1$; thus, keeping terms up to the first order, we have

$$
\rho(\mathbf{r}) / \varepsilon \cong-\frac{e^{2} n_{\infty}}{k_{\mathrm{B}} \varepsilon}\left(\frac{1}{T_{i}}+\frac{1}{T_{e}}\right) \cdot \Phi(\mathbf{r})=-\frac{1}{\lambda_{\mathrm{D} \infty}^{2}} \cdot \Phi(\mathbf{r}),
$$

and the Poisson equation of Gauss' law of electrodynamics is linearized

$$
\nabla^{2} \Phi(\mathbf{r})=-\rho(\mathbf{r}) / \varepsilon \cong \frac{1}{\lambda_{\mathrm{D}_{\infty}}^{2}} \cdot \Phi(\mathbf{r})
$$


We now examine the three different dimensionalities of the electric field/potential symmetry.

\section{B.1. 1-D potential: planar charge perturbation}

Let the planar charge perturbation be at the $y-z$ plane. The 1-D Poisson equation along $x$-axis is written as

$$
\Phi^{\prime \prime}(x)=-\rho(x) / \varepsilon \cong \frac{1}{\lambda_{\mathrm{D}_{\infty}}^{2}} \cdot \Phi(x),
$$

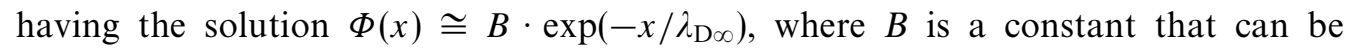
expressed in terms of the surface charge density $\sigma$; this is related to the electric field at $x=0$, i.e. $\frac{1}{2}(\sigma / \varepsilon)=E(x=0)=-\Phi^{\prime}(x=0) \cong B / \lambda_{\mathrm{D} \infty}$, or $B \cong \frac{1}{2}(\sigma / \varepsilon) \lambda_{\mathrm{D} \infty}$; hence, the solution becomes

$$
\Phi(x) \cong \frac{\sigma}{2 \varepsilon} \lambda_{\mathrm{D} \infty} \cdot \exp \left(-x / \lambda_{\mathrm{D} \infty}\right)
$$

Equation (B4) describes the potential along the direction $x$ that is perpendicular to the planar charge perturbation of surface density $\sigma$; this is given by $\sigma=$ $2\left(\Delta N / N_{\mathrm{D}}\right) e n_{\infty} \lambda_{\mathrm{D} \infty}$, hence,

$$
\begin{gathered}
\Phi(x) \cong\left(\Delta N / N_{\mathrm{D}}\right) e \varepsilon^{-1} n_{\infty} \lambda_{\mathrm{D} \infty}^{2} \cdot \exp \left(-x / \lambda_{\mathrm{D} \infty}\right), \text { or } \\
R(x) \equiv \frac{N_{\mathrm{D}} e \Phi(x)}{\Delta N \frac{1}{2} k_{\mathrm{B}} T_{0}} \cong 2 \cdot \exp \left(-x / \lambda_{\mathrm{D} \infty}\right) .
\end{gathered}
$$

Note that (B5b) holds for $x \geqslant 0$, and can written as $R(x) \cong 2 \cdot \exp \left(-|x| / \lambda_{\mathrm{D} \infty}\right)$ for any $x$. In addition, the ratio of the shielded to the unshielded potential is

$$
\Phi(x) / \Phi(0) \cong \exp \left(-x / \lambda_{\mathrm{D} \infty}\right) .
$$

Therefore, the Debye length is determined by the two interpretations,

$$
\text { (1) } R\left(\lambda_{\mathrm{D} \infty}\right)=2 \cdot(1 / \mathrm{e}) \text {, and }(2) \Phi\left(\lambda_{\mathrm{D} \infty}\right) / \Phi(0) \cong 1 / \mathrm{e}
$$

that correspond to the two interpretations of the Debye length, given in (5a) and (5b), respectively.

\section{B.2. 2-D potential: linear charge perturbation}

In the case of a linear charge perturbation of large length $L$ (practically, at least $L \gg \lambda_{\mathrm{D}}$ ) along the $z$-axis, the symmetry of the electric potential and field is cylindrical. The 2-D Poisson equation along $x-y$ plane is isotropic and written in terms of the cylindrical radius $\rho=\sqrt{x^{2}+y^{2}}$,

$$
\frac{1}{\rho} \frac{d}{d \rho}\left[\rho \frac{d \Phi(\rho)}{d \rho}\right]=-\rho(\rho) / \varepsilon \cong \frac{1}{\lambda_{\mathrm{D} \infty}^{2}} \cdot \Phi(\rho),
$$

that is given by the Bessel differential equation

$$
\Phi^{\prime \prime}(\rho)+\frac{1}{\rho} \Phi^{\prime}(\rho)-\frac{1}{\lambda_{\mathrm{D} \infty}^{2}} \cdot \Phi(\rho)=0,
$$


with a solution given by the bounded modified Bessel function of the second kind, $\Phi(\rho)=B \cdot K_{0}\left(\rho / \lambda_{\mathrm{D} \infty}\right)$. The function $K_{0}(x)$ has the asymptotic behavior,

$$
K_{0}(x) \cong \begin{cases}\frac{\sqrt{\pi}}{2} \cdot \frac{\exp (-x)}{\sqrt{x}}, & x \gg 1, \\ \frac{\exp (-x)}{\sqrt{x}}, & x \sim O(1), \\ -\ln (x / 2)-\gamma, & x \ll 1 .\end{cases}
$$

$(\gamma=0.5772156649 \ldots$ is the Euler constant; the asymptotic behavior for large $x$ is sufficiently accurate $(\sim 90 \%)$ even for $x \sim 1$.) In order to determine the constant $B$, we find the electric field for distances close to perturbation, $\rho / \lambda_{\mathrm{D} \infty} \ll 1$; the potential is written as $\Phi(\rho) \cong-B \cdot \ln (\rho)$, where we ignore the constant quantity $B \cdot \ln \left(2 \lambda_{\text {D }}\right)-\gamma$. Hence, the corresponding electric field is $E(\rho) \cong B / \rho$; comparing this with the electric field of a linear charge perturbation of large length, $E(\rho) \cong l /(2 \pi \varepsilon \rho)$ with $l$ noting the linear charge density (e.g. see Dash and Khuntia 2010), we obtain that $B=l /(2 \pi \varepsilon)$. Hence,

$$
\Phi(\rho) \cong \frac{l}{2 \pi \varepsilon} \cdot K_{0}\left(\rho / \lambda_{\mathrm{D} \infty}\right) \cong \frac{l}{2 \pi \varepsilon} \cdot \begin{cases}\sqrt{\frac{\pi}{2}} \cdot \frac{\exp \left(-\rho / \lambda_{\mathrm{D} \infty}\right)}{\sqrt{\rho / \lambda_{\mathrm{D} \infty}}}, & \rho \gg \lambda_{\mathrm{D} \infty}, \\ \frac{2}{\sqrt{\pi}} \cdot \frac{\operatorname{exp(-\rho /\lambda _{\mathrm {D}\infty })}}{\sqrt{\rho / \lambda_{\mathrm{D} \infty}},}, & \rho \sim O\left(\lambda_{\mathrm{D} \infty}\right), \\ \ln \left(\lambda_{\mathrm{D} \infty} / \rho\right)+\text { constant }, & \rho \ll \lambda_{\mathrm{D} \infty} .\end{cases}
$$

The linear charge density is equal to $l=\left(\Delta N / N_{\mathrm{D}}\right) e n_{\infty} \pi \lambda_{\mathrm{D} \infty}^{2}$, hence,

$$
\begin{gathered}
\Phi(\rho) \cong\left(\Delta N / N_{\mathrm{D}}\right) \frac{1}{2} \frac{2}{\sqrt{\pi}} e \varepsilon^{-1} n_{\infty} \lambda_{\mathrm{D} \infty}^{5 / 2} \cdot \exp \left(-\rho / \lambda_{\mathrm{D} \infty}\right) / \sqrt{\rho}, \text { or } \\
R(\rho) \equiv \frac{N_{\mathrm{D}} e \Phi(\rho)}{\Delta N \frac{1}{2} k_{\mathrm{B}} T_{0}} \cong \exp \left(-\rho / \lambda_{\mathrm{D} \infty}\right) / \sqrt{\rho / \lambda_{\mathrm{D} \infty}} .
\end{gathered}
$$

The potential is also written as

$$
\Phi(\rho) / \Phi(\rho \rightarrow 0) \cong \exp \left(-\rho / \lambda_{\mathrm{D} \infty}\right) .
$$

Therefore, the Debye length is determined by

$$
\text { (1) } R\left(\lambda_{\mathrm{D} \infty}\right)=(1 / \mathrm{e}) \text { and }(2) \Phi\left(\lambda_{\mathrm{D} \infty}\right) / \Phi(\rho \rightarrow 0) \cong 1 / \mathrm{e}
$$

that correspond to the two interpretations of the Debye length, given in (5a) and (5b), respectively.

\section{B.3. 3-D potential: point-charge perturbation}

In the case of a point charge perturbation $Q$, the symmetry of the electric potential and field is spherical. The 3-D Poisson equation is isotropic and written in terms only of the spherical radius $r=\sqrt{x^{2}+y^{2}+z^{2}}$,

$$
\frac{1}{r^{2}} \frac{d}{d r}\left[r^{2} \frac{d \Phi(r)}{d r}\right]=-\rho(r) / \varepsilon \cong \frac{1}{\lambda_{\mathrm{D} \infty}^{2}} \cdot \Phi(r),
$$

that is given by the differential equation

$$
\Phi^{\prime \prime}(r)+\frac{2}{r} \Phi^{\prime}(r)-\frac{1}{\lambda_{\mathrm{D} \infty}^{2}} \cdot \Phi(r)=0,
$$

with the solution given by the potential $\Phi(r)=B \exp \left(-r / \lambda_{\mathrm{D} \infty}\right) / r$ (called Yukawa or Debye-Hückel). For $r \rightarrow 0$, this describes the potential of the charge $Q$ without the 
plasma shielding, i.e. $\Phi(r \rightarrow 0)=Q /(4 \pi \varepsilon r)=B / r$, or

$$
\Phi(r) \cong \frac{Q}{4 \pi \varepsilon} \cdot \frac{1}{r} \exp \left(-r / \lambda_{\mathrm{D} \infty}\right) .
$$

The point charge is written as $Q=\left(\Delta N / N_{\mathrm{D}}\right) e n_{\infty}(4 \pi / 3) \lambda_{\mathrm{D} \infty}^{3}$, hence,

$$
\begin{gathered}
\Phi(r) \cong\left(\Delta N / N_{\mathrm{D}}\right) \frac{1}{3} e \varepsilon^{-1} n_{\infty} \lambda_{\mathrm{D} \infty}^{3} \cdot \exp \left(-r / \lambda_{\mathrm{D} \infty}\right) / r, \text { or } \\
R(r) \equiv \frac{N_{\mathrm{D}} e \Phi(r)}{\Delta N \frac{1}{2} k_{\mathrm{B}} T_{0}} \cong(2 / 3) \cdot \frac{\lambda_{\mathrm{D} \infty}}{r} \cdot \exp \left(-r / \lambda_{\mathrm{D} \infty}\right) .
\end{gathered}
$$

The potential is also written as

$$
\Phi(r) / \Phi(r \rightarrow 0) \cong \exp \left(-r / \lambda_{\mathrm{D} \infty}\right) .
$$

Now the Debye length determines the distance for which

$$
\text { (1) } R\left(\lambda_{\mathrm{D} \infty}\right)=(2 / 3) \cdot(1 / \mathrm{e}) \text { and }(2) \Phi\left(\lambda_{\mathrm{D} \infty}\right) / \Phi(r \rightarrow 0) \cong 1 / \mathrm{e}
$$

that correspond to the two Debye length interpretations, given in (5a) and (5b).

\section{Appendix C. Debye length in non-equilibrium plasmas}

For plasmas in stationary states out of thermal equilibrium, the ion and electron densities are described by kappa distributions, which are written in terms of the invariant kappa index $\kappa_{0}$. Namely,

$$
\begin{aligned}
& n_{i}=n_{\infty} \cdot\left\{1+\frac{e\left[\Phi(\mathbf{r})-\Phi_{\infty}\right]}{\kappa_{0} k_{\mathrm{B}} T_{i}-e\left(\bar{\Phi}_{i}-\Phi_{\infty}\right)}\right\}^{-\kappa_{0}-1}, \\
& n_{e}=n_{\infty} \cdot\left\{1-\frac{e\left[\Phi(\mathbf{r})-\Phi_{\infty}\right]}{\kappa_{0} k_{\mathrm{B}} T_{e}+e\left(\bar{\Phi}_{e}-\Phi_{\infty}\right)}\right\}^{-\kappa_{0}-1} .
\end{aligned}
$$

Considering zero potential at large distances from the perturbation, $\Phi_{\infty} \cong 0$, we have

$$
n_{i}=n_{\infty} \cdot\left[1+\frac{e \Phi(\mathbf{r})}{\kappa_{0} k_{\mathrm{B}} T_{i}-e \bar{\Phi}_{i}}\right]^{-\kappa_{0}-1}, n_{e}=n_{\infty} \cdot\left[1-\frac{e \Phi(\mathbf{r})}{\kappa_{0} k_{\mathrm{B}} T_{e}+e \bar{\Phi}_{e}}\right]^{-\kappa_{0}-1} .
$$

The total charge density is $\rho=e\left(n_{i}-n_{e}\right)$, which is expanded in terms of the ratio of the potential to thermal energy. The kappa index is involved in the expansion with a rational function (that tends to 1 for $\kappa_{0} \rightarrow \infty$ ). The first term is a good approximation of the expansion, if $e \Phi(\mathbf{r}) /\left(\frac{\kappa_{0}}{\kappa_{0}+1} k_{\mathrm{B}} T_{0}\right) \ll 1$. Indeed, the densities are written as

$$
\begin{aligned}
& n_{i}=n_{\infty}\left\{1-\frac{e\left[\bar{\Phi}_{i}-\Phi_{\infty}\right]}{\kappa_{0} k_{\mathrm{B}} T_{i}}\right\}^{\kappa_{0}+1} \cdot\left\{1+\frac{e\left[\Phi(\mathbf{r})-\bar{\Phi}_{i}\right]}{\kappa_{0} k_{\mathrm{B}} T_{i}}\right\}^{-\kappa_{0}-1}, \\
& n_{e}=n_{\infty} \cdot\left\{1-\frac{e\left[\Phi(\mathbf{r})-\Phi_{\infty}\right]}{\kappa_{0} k_{\mathrm{B}} T_{e}+e\left(\bar{\Phi}_{e}-\Phi_{\infty}\right)}\right\}^{-\kappa_{0}-1},
\end{aligned}
$$

that are expanded as

$$
\begin{aligned}
& n_{i}=n_{\infty}\left\{1-\frac{\kappa_{0}+1}{\kappa_{0}} \cdot \frac{e\left[\bar{\Phi}_{i}-\Phi_{\infty}\right]}{k_{\mathrm{B}} T_{i}}+\ldots\right\} \cdot\left\{1-\frac{\kappa_{0}+1}{\kappa_{0}} \cdot \frac{e\left[\Phi(\mathbf{r})-\bar{\Phi}_{i}\right]}{k_{\mathrm{B}} T_{i}}+\ldots\right\}, \\
& n_{e}=n_{\infty}\left\{1+\frac{\kappa_{0}+1}{\kappa_{0}} \cdot \frac{e\left[\bar{\Phi}_{e}-\Phi_{\infty}\right]}{k_{\mathrm{B}} T_{e}}+\ldots\right\} \cdot\left\{1+\frac{\kappa_{0}+1}{\kappa_{0}} \cdot \frac{e\left[\Phi(\mathbf{r})-\bar{\Phi}_{e}\right]}{k_{\mathrm{B}} T_{e}}+\ldots\right\},
\end{aligned}
$$


or,

$$
\begin{aligned}
& n_{i}=n_{\infty}\left[1-\frac{\kappa_{0}+1}{\kappa_{0}} \cdot \frac{e \Phi(\mathbf{r})}{k_{\mathrm{B}} T_{i}}\right]+O\left[\left(\frac{\kappa_{0}+1}{\kappa_{0}}\right) \frac{e \Phi(\mathbf{r})}{k_{\mathrm{B}} T_{0}}\right]^{2}, \\
& n_{e}=n_{\infty}\left[1+\frac{\kappa_{0}+1}{\kappa_{0}} \cdot \frac{e \Phi(\mathbf{r})}{k_{\mathrm{B}} T_{e}}\right]+O\left[\left(\frac{\kappa_{0}+1}{\kappa_{0}}\right) \frac{e \Phi(\mathbf{r})}{k_{\mathrm{B}} T_{0}}\right]^{2},
\end{aligned}
$$

where we take also into account that $\Phi_{\infty} \cong 0$. Then, the density of the excess of electrons is given by

$$
n_{e}-n_{i}=\frac{\kappa_{0}+1}{\kappa_{0}} \cdot \frac{e \Phi(\mathbf{r})}{k_{\mathrm{B}} T_{0}}+O\left[\left(\frac{\kappa_{0}+1}{\kappa_{0}}\right) \frac{e \Phi(\mathbf{r})}{k_{\mathrm{B}} T_{0}}\right]^{2}
$$

which is a good approximation if

$$
e \Phi(\mathbf{r}) /\left(\frac{\kappa_{0}}{\kappa_{0}+1} k_{\mathrm{B}} T_{0}\right) \ll 1
$$

The linearization of the density gives

$$
\rho(\mathbf{r}) / \varepsilon \cong-\frac{e^{2} n_{\infty}}{k_{\mathrm{B}} \varepsilon}\left(\frac{1}{T_{i}}+\frac{1}{T_{e}}\right) \cdot \frac{\kappa_{0}+1}{\kappa_{0}} \cdot \Phi(\mathbf{r})=-\frac{1}{\lambda_{\mathrm{D}}^{2}} \cdot \frac{\kappa_{0}+1}{\kappa_{0}} \cdot \Phi(\mathbf{r})=-\frac{1}{\lambda_{\mathrm{D},{ }_{\mathrm{K}}}^{2}} \cdot \Phi(\mathbf{r})
$$

and the linearized Poisson equation is

$$
\nabla^{2} \Phi(\mathbf{r})=-\rho(\mathbf{r}) / \varepsilon \cong \frac{1}{\lambda_{\mathrm{D}, \mathrm{K}}^{2}} \cdot \Phi(\mathbf{r})
$$

We now consider the same three dimensionality cases for plasmas out of thermal equilibrium.

\section{C.1. 1-D potential: planar charge perturbation}

The Poisson equation along the $x$-axis is

$$
\Phi^{\prime \prime}(x)=-\rho(x) / \varepsilon \cong \frac{1}{\lambda_{\mathrm{D}, \mathrm{K}}^{2}} \cdot \Phi(x)
$$

having the solution

$$
\Phi(x) \cong \frac{\sigma}{2 \varepsilon} \lambda_{\mathrm{D},{ }_{\mathrm{K}}} \cdot \exp \left(-x / \lambda_{\mathrm{D}, \mathrm{K}}\right) .
$$

At this point we consider the exact dependence of the Debye length on the kappa index to be unknown and symbolized with $\lambda_{\mathrm{D},{ }_{A}}$ as in (7). Hence, the surface density $\sigma$ is given by $\sigma=2\left(\Delta N / N_{\mathrm{D}}\right) e n_{\infty} \lambda_{\mathrm{D},{ }_{\Lambda}}$, and the potential is written as

$$
\Phi(x) \cong\left(\Delta N / N_{\mathrm{D}}\right) e \varepsilon^{-1} n_{\infty} \lambda_{\mathrm{D}, \mathrm{K}} \lambda_{\mathrm{D},{ }_{\Lambda}} \cdot \exp \left(-x / \lambda_{\mathrm{D}, \mathrm{K}}\right) .
$$

Then, we have

$$
R(x) \equiv \frac{N_{\mathrm{D}} e \Phi(x)}{\Delta N \frac{1}{2} k_{\mathrm{B}} T_{0}} \cong 2 \cdot \frac{\lambda_{\mathrm{D}, \mathrm{K}} \lambda_{\mathrm{D}, \Lambda}}{\lambda_{\mathrm{D} \infty}^{2}} \cdot \exp \left(-x / \lambda_{\mathrm{D}, \mathrm{K}}\right) .
$$

The potential is also written as

$$
\Phi(x) / \Phi(0) \cong \exp \left(-x / \lambda_{\mathrm{D}, \mathrm{K}}\right)
$$

and at the Debye length the two interpretations, $(5 \mathrm{a})$ and $(5 \mathrm{~b})$, become

$$
\begin{aligned}
& \text { (1) } R\left(\lambda_{\mathrm{D}, \Lambda}\right)=2 \cdot\left(\lambda_{\mathrm{D}, \mathrm{K}} \lambda_{\mathrm{D}, \Lambda} / \lambda_{\mathrm{D} \infty}^{2}\right) \cdot \exp \left(-\lambda_{\mathrm{D}, \Lambda} / \lambda_{\mathrm{D}, \mathrm{K}}\right) \text { and } \\
& \text { (2) } \Phi\left(\lambda_{\mathrm{D}, \Lambda}\right) / \Phi(0) \cong \exp \left(-\lambda_{\mathrm{D}, \Lambda} / \lambda_{\mathrm{D}, \mathrm{K}}\right)
\end{aligned}
$$


Typically, the Debye length in non-equilibrium plasmas is taken to be $\lambda_{\mathrm{D}, \mathrm{K}}$ (i.e. $\left.\lambda_{\mathrm{D}, \Lambda}=\lambda_{\mathrm{D}, \mathrm{K}}\right)$, because of Interpretation 2 in $(5 \mathrm{~b})$, that is, $\Phi\left(\lambda_{\mathrm{D}, \mathrm{K}}\right) / \Phi(0) \cong 1 / \mathrm{e}$. However, Interpretation 1 is quite different; it cannot be equal to $1 / \mathrm{e}$ or any other constant value, independent of the kappa index (see Sec. 4).

\section{C.2. 2-D potential: linear charge perturbation}

The Poisson equation for a linear charge perturbation that applies cylindrical symmetry is written as

$$
\Phi^{\prime \prime}(\rho)+\frac{1}{\rho} \Phi^{\prime}(\rho)-\frac{1}{\lambda_{\mathrm{D}, \mathrm{K}}^{2}} \cdot \Phi(\rho)=0,
$$

with a solution given by

$$
\Phi(\rho) \cong \frac{l}{2 \pi \varepsilon} \cdot K_{0}\left(\rho / \lambda_{\mathrm{D}, \mathrm{K}}\right) \cong \frac{l}{2 \pi \varepsilon} \cdot \begin{cases}\sqrt{\frac{\pi}{2}} \cdot \frac{\exp \left(-\rho / \lambda_{\mathrm{D}, \mathrm{K}}\right)}{\sqrt{\rho / \lambda_{\mathrm{D}, \mathrm{K}}}}, & \rho \gg \lambda_{\mathrm{D}, \mathrm{K}}, \\ \sqrt{\frac{2}{\pi}} \cdot \frac{\exp \left(-\rho / \lambda_{\mathrm{D}, \mathrm{K}}\right)}{\sqrt{\rho / \lambda_{\mathrm{D}, \mathrm{K}}}}, & \rho \sim O\left(\lambda_{\mathrm{D}, \mathrm{K}}\right), \\ \ln \left(\lambda_{\mathrm{D}, \mathrm{K}} / \rho\right)+\text { constant }, & \rho \ll \lambda_{\mathrm{D}, \mathrm{K}} .\end{cases}
$$

The linear charge density is given by $l=\left(\Delta N / N_{\mathrm{D}}\right) e n_{\infty} \pi \lambda_{\mathrm{D}, \Lambda}^{2}$, hence,

$$
\begin{gathered}
\Phi(\rho) \cong\left(\Delta N / N_{\mathrm{D}}\right) \frac{1}{2} \sqrt{\frac{2}{\pi}} e \varepsilon^{-1} n_{\infty} \lambda_{\mathrm{D}, \Lambda}^{2} \sqrt{\lambda_{\mathrm{D}, \mathrm{K}}} \cdot \exp \left(-\rho / \lambda_{\mathrm{D}, \mathrm{K}}\right) / \sqrt{\rho}, \text { or } \\
R(\rho) \equiv \frac{N_{\mathrm{D}} e \Phi(\rho)}{\Delta N \frac{1}{2} k_{\mathrm{B}} T_{0}} \cong\left(\lambda_{\mathrm{D}, \Lambda} / \lambda_{\mathrm{D} \infty}\right)^{2} \cdot \exp \left(-\rho / \lambda_{\mathrm{D}, \mathrm{K}}\right) / \sqrt{\rho / \lambda_{\mathrm{D}, \mathrm{K}}}
\end{gathered}
$$

The potential is also written as

$$
\Phi(\rho) / \Phi(\rho \rightarrow 0) \cong \exp \left(-\rho / \lambda_{\mathrm{D}, \mathrm{K}}\right) .
$$

Therefore, the Debye length is determined by

$$
\begin{aligned}
& \text { (1) } R\left(\lambda_{\mathrm{D}, \Lambda}\right)=\left(\lambda_{\mathrm{D}, \Lambda}^{3 / 2} \lambda_{\mathrm{D}, \mathrm{K}}^{1 / 2} / \lambda_{\mathrm{D} \infty}^{2}\right) \cdot \exp \left(-\lambda_{\mathrm{D}, \Lambda} / \lambda_{\mathrm{D}, \mathrm{K}}\right) \text { and } \\
& \text { (2) } \Phi\left(\lambda_{\mathrm{D}, \Lambda}\right) / \Phi(\rho \rightarrow 0) \cong \exp \left(-\lambda_{\mathrm{D}, \Lambda} / \lambda_{\mathrm{D}, \mathrm{K}}\right)
\end{aligned}
$$

that correspond to the two interpretations of the Debye length, given in (5a) and (5b), respectively.

\section{C.3. 3-D potential: point-charge perturbation}

For a point charge perturbation $Q$, there is a spherical symmetry and the Poisson differential equation is

$$
\Phi^{\prime \prime}(r)+\frac{2}{r} \Phi^{\prime}(r)-\frac{1}{\lambda_{\mathrm{D}, \mathrm{K}}^{2}} \cdot \Phi(r)=0,
$$

with the solution given by

$$
\Phi(r) \cong \frac{Q}{4 \pi \varepsilon} \cdot \frac{1}{r} \exp \left(-r / \lambda_{\mathrm{D}, \mathrm{K}}\right) .
$$

The point charge is written as $Q=\left(\Delta N / N_{\mathrm{D}}\right) e n_{\infty}(4 \pi / 3) \lambda_{\mathrm{D}, \Lambda}^{3}$, hence,

$$
\begin{array}{r}
\Phi(r) \cong\left(\Delta N / N_{\mathrm{D}}\right) \frac{1}{3} e \varepsilon^{-1} n_{\infty} \lambda_{\mathrm{D}, \Lambda}^{3} \cdot \exp \left(-r / \lambda_{\mathrm{D}, \mathrm{K}}\right) / r, \text { or }, \\
R(r) \equiv \frac{N_{\mathrm{D}} e \Phi(r)}{\Delta N \frac{1}{2} k_{\mathrm{B}} T_{0}} \cong(2 / 3) \cdot\left(\lambda_{\mathrm{D}, \Lambda} / \lambda_{\mathrm{D} \infty}\right)^{3} \cdot \frac{\lambda_{\mathrm{D} \infty}}{r} \exp \left(-r / \lambda_{\mathrm{D}, \mathrm{K}}\right) .
\end{array}
$$


The potential is also written as

$$
\Phi(r) / \Phi(r \rightarrow 0) \cong \exp \left(-r / \lambda_{\mathrm{D}, \mathrm{K}}\right) .
$$

Now the Debye length determines the distance for which

$$
\begin{aligned}
& \text { (1) } R\left(\lambda_{\mathrm{D}}\right)=(2 / 3) \cdot\left(\lambda_{\mathrm{D}, \Lambda} \lambda_{\mathrm{D} \infty}\right)^{2} \cdot \exp \left(-\lambda_{\mathrm{D}, \Lambda} / \lambda_{\mathrm{D}, \mathrm{K}}\right) \text { and } \\
& \text { (2) } \Phi\left(\lambda_{\mathrm{D}, \Lambda}\right) / \Phi(r \rightarrow 0) \cong \exp \left(-\lambda_{\mathrm{D}, \Lambda} \lambda_{\mathrm{D}_{,}}\right)
\end{aligned}
$$

that correspond to the two Debye length interpretations, given in (5a) and (5b).

\section{Appendix D. Excess of electrons due to perturbation}

In this Appendix, we show that the number of singly charged ions $\Delta N_{\mathrm{CP}}$ that contribute to the charge perturbation is equal to the number of excess electrons $\Delta N$ in the plasma. This is required for the neutrality of the plasma. We show this in general, and verify it specifically for the three dimensionalities $d=1,2,3$ (Fig. 1).

The density of the electron excess $n_{e}-n_{i}$ is integrated over all the space to give the number of excess electrons $\Delta N$, i.e.

$$
\Delta N=\int_{\mathbf{r} \in V}\left[n_{e}(\mathbf{r})-n_{i}(\mathbf{r})\right] d V,
$$

while the density of the ions that contribute to the charge perturbation $n_{\mathrm{CP}}$ is integrated over all the space to give the number of singly charged ions $\Delta N_{\mathrm{CP}}$, i.e.

$$
\Delta N_{\mathrm{CP}}=\int_{\mathbf{r} \in V} n_{C P}(\mathbf{r}) d V .
$$

The elementary volume $d V$ and the position vector $\mathbf{r}$ span the whole volume $V$. Gauss' integrated law of electrodynamics gives

$$
\int_{\mathbf{r} \in S=\partial V} \mathbf{E} \cdot d \mathbf{S}=\frac{1}{\varepsilon} \int_{\mathbf{r} \in V} \rho_{\text {all }}(\mathbf{r}) d V
$$

where the integration is on the surface-boundary of the volume $V$, denoted with $S=\partial V$. The total charge distribution density $\rho_{\text {all }}$ includes both the density of the ions that contribute to the charge perturbation $n_{\mathrm{CP}}$ and the density of the excess electrons $n_{e}-n_{i}$, so that

$$
\int_{\mathbf{r} \in S=\partial V} \mathbf{E} \cdot d \mathbf{S}=\frac{e}{\varepsilon} \int_{\mathbf{r} \in V}\left[n_{\mathrm{CP}}(\mathbf{r})-n_{e}(\mathbf{r})+n_{i}(\mathbf{r})\right] d V=\frac{e}{\varepsilon} \cdot\left[\Delta N_{\mathrm{CP}}-\Delta N(V)\right],
$$

where $\Delta N(V)$ denotes the number of the excess electrons within a volume $V$. At large distances from the perturbation, $r \rightarrow \infty, V \rightarrow \infty$, the electrostatic potential and field tend to zero, $\Phi \rightarrow 0, \mathbf{E} \rightarrow 0$, and $\Delta N(V)$ gives the whole number of excess electrons. Then, (D3) becomes

$$
\lim _{r \rightarrow \infty} \int_{\mathbf{r} \in S=\partial V} \vec{\nabla} \Phi(\mathbf{r}) \cdot d \mathbf{S}=\frac{e}{\varepsilon} \cdot\left[\Delta N-\Delta N_{\mathrm{CP}}\right] \rightarrow 0,
$$

that is, the number of excess electrons (from all the plasma) equals the number of ions that constitute the charge perturbation. This can be easily verified for the three 
dimensionalities $d=1,2,3$ (Fig. 1): By substituting $n_{e}-n_{i}$ in (D1a), as given in (C6a), we derive

$$
\Delta N=\int_{\mathbf{r} \in V}\left[n_{e}(\mathbf{r})-n_{i}(\mathbf{r})\right] d V=n_{\infty} \cdot \int_{\mathbf{r} \in V} \frac{\kappa_{0}+1}{\kappa_{0}} \cdot \frac{e \Phi(\mathbf{r})}{k_{B} T_{0}} d V,
$$

where the elementary volume of the 3-D plasma, $d V$, under the $d$-D geometry is given by (1) $A \cdot 2 d r$ for $d=1$, (2) $L \cdot 2 \pi r d r$ for $d=2$, and (3) $4 \pi r^{2} d r$ for $d=3$; all three written in a compact way as $L^{3-d} \cdot B_{d} r^{d-1} d r$, where we wrote $A=L^{2} ; B_{d}$ is the surface of a $d$-D sphere of unit radius given by $B_{d}=2 \pi^{\frac{d}{2}} / \Gamma\left(\frac{d}{2}\right)$. We also substitute the potential expression (Table 2),

$$
\frac{e \Phi(\mathbf{r})}{k_{\mathrm{B}} T_{0}}=\left[\frac{d}{2} \Gamma\left(\frac{d+1}{2}\right)\right]^{-1} \cdot \frac{\Delta N_{\mathrm{CP}}}{N_{\mathrm{D}}} \cdot \Lambda\left(\kappa_{0}\right)^{d}\left(\frac{\kappa_{0}}{\kappa_{0}+1}\right)^{(2-d) / 2} \cdot \frac{\exp \left(-r / \lambda_{\mathrm{D}, \mathrm{K}}\right)}{\left(r / \lambda_{\mathrm{D}, \mathrm{K}}\right)^{(d-1) / 2}},
$$

where

$$
\begin{aligned}
& \frac{\Delta N_{\mathrm{CP}}}{N_{\mathrm{D}}}(d=1)=\frac{\sigma}{2 e n_{\infty} \lambda_{\mathrm{D}, \Lambda}}, \frac{\Delta N_{\mathrm{CP}}}{N_{D}}(d=2)=\frac{l}{e n_{\infty} \pi \lambda_{\mathrm{D},{ }_{\Lambda}}^{2}}, \\
& \frac{\Delta N_{\mathrm{CP}}}{N_{\mathrm{D}}}(d=3)=\frac{Q}{e n_{\infty}(4 \pi / 3) \lambda_{\mathrm{D},{ }_{\Lambda}}^{3}} .
\end{aligned}
$$

Hence, (D5) becomes

$$
\begin{gathered}
\Delta N=\Delta N_{\mathrm{CP}} \cdot\left[d \cdot \Gamma\left(\frac{d+1}{2}\right)\right]^{-1} \cdot\left[\Lambda\left(\kappa_{0}\right) / \sqrt{\frac{\kappa_{0}}{\kappa_{0}+1}}\right]^{d} \cdot \frac{n_{\infty}}{N_{\mathrm{D}}} \cdot \int_{\mathrm{r} \in V} \frac{\exp \left(-r / \lambda_{\mathrm{D}, \mathrm{K}}\right)}{\left(r / \lambda_{\mathrm{D}, \mathrm{K}}\right)^{(d-1) / 2}} d V, \text { or, } \\
\Delta N=\Delta N_{\mathrm{CP}} \cdot \Gamma\left(\frac{d+1}{2}\right)^{-1} \cdot \frac{n_{\infty} \cdot L^{3-d}\left(B_{d} / d\right) \lambda_{\mathrm{D}, \Lambda}^{d}}{N_{\mathrm{D}}} \cdot \int_{0}^{\infty} \frac{\exp (-\xi)}{\xi^{(d-1) / 2}} \xi^{d-1} d \xi,
\end{gathered}
$$

where we set $\xi \equiv r / \lambda_{\mathrm{D}, \mathrm{K}}, \lambda_{\mathrm{D}, \Lambda}=\left[\Lambda\left(\kappa_{0}\right) / \sqrt{\frac{\kappa_{0}}{\kappa_{0}+1}}\right] \cdot \lambda_{\mathrm{D}, \mathrm{K}}$. The Debye volume is $L^{3-d}\left(B_{d} / d\right) \lambda_{\mathrm{D}, \Lambda}^{d}$, so that $N_{\mathrm{D}}=n_{\infty} \cdot L^{3-d}\left(B_{d} / d\right) \lambda_{\mathrm{D}, \Lambda}^{d}$; the elementary volume is $d V=\left(B_{d} / d\right) L^{3-d} r^{d-1} d r$. Hence,

$$
\Delta N=\Delta N_{\mathrm{CP}} \cdot \Gamma\left(\frac{d+1}{2}\right)^{-1} \cdot \int_{0}^{\infty} \exp (-\xi) \xi^{(d-1) / 2} d \xi .
$$

Finally, since $\int_{0}^{\infty} \exp (-\xi) \xi^{(d+1) / 2-1} d \xi=\Gamma\left(\frac{d+1}{2}\right)$, we end up with

$$
\Delta N=\Delta N_{\mathrm{CP}} \text {. }
$$

\section{Appendix E. Large perturbations}

A better approximation of the potential energy $e \Phi$ and its ratio with the thermal energy $R$ is obtained by using density functions without the small potential approximation $e \Phi(r) \ll \kappa_{0} k_{\mathrm{B}} T_{i, e}$. Given the ion/electron densities

$$
n_{i}=n_{\infty} \cdot\left[1+\frac{e \Phi(\mathbf{r})}{\kappa_{0} k_{\mathrm{B}} T_{i}-e \bar{\Phi}_{i}}\right]^{-\kappa_{0}-1}, n_{e}=n_{\infty} \cdot\left[1-\frac{e \Phi(\mathbf{r})}{\kappa_{0} k_{\mathrm{B}} T_{e}+e \bar{\Phi}_{e}}\right]^{-\kappa_{0}-1},
$$


integrated within a Debye length $\lambda_{\mathrm{D}, \Lambda}$,

$$
\begin{aligned}
& \frac{N_{i}}{N_{\mathrm{D}}}=1-\frac{\Delta N_{i}}{N_{\mathrm{D}}}=\frac{n_{\infty}}{N_{\mathrm{D}}} \cdot \int_{0}^{\lambda_{\mathrm{D}, \Lambda}}\left[1+\frac{e \Phi(r)}{\kappa_{0} k_{\mathrm{B}} T_{i}-e \bar{\Phi}_{i}}\right]^{-\kappa_{0}-1} 4 \pi r^{2} d r \cong\left[1+\frac{e \tilde{\Phi}\left(\lambda_{\mathrm{D}, \Lambda}\right)}{\kappa_{0} k_{\mathrm{B}} T_{i}}\right]^{-\kappa_{0}-1}, \\
& \frac{N_{e}}{N_{\mathrm{D}}}=1+\frac{\Delta N_{e}}{N_{\mathrm{D}}}=\frac{n_{\infty}}{N_{\mathrm{D}}} \cdot \int_{0}^{\lambda_{\mathrm{D}, \Lambda}}\left[1-\frac{e \Phi(r)}{\kappa_{0} k_{\mathrm{B}} T_{e}+e \bar{\Phi}_{e}}\right]^{-\kappa_{0}-1} 4 \pi r^{2} d r \cong\left[1-\frac{e \tilde{\Phi}\left(\lambda_{\mathrm{D}, \Lambda}\right)}{\kappa_{0} k_{\mathrm{B}} T_{e}}\right]^{-\kappa_{0}-1},
\end{aligned}
$$

we obtain

$$
\frac{e \tilde{\Phi}\left(\lambda_{\mathrm{D}, \Lambda}\right)}{k_{\mathrm{B}} T_{0}} \sim \kappa_{0} \cdot\left[\left(1-\frac{\Delta N_{i}}{N_{\mathrm{D}}}\right)^{-\frac{1}{\kappa_{0}+1}}-\left(1+\frac{\Delta N_{e}}{N_{\mathrm{D}}}\right)^{-\frac{1}{\kappa_{0}+1}}\right],
$$

where the effective temperature is modified to $T_{0}=\left[\left(T_{i}-e \bar{\Phi}_{i} / \kappa_{0} k_{\mathrm{B}}\right)^{-1}+\left(T_{e}+\right.\right.$ $\left.\left.e \bar{\Phi}_{e} / \kappa_{0} k_{\mathrm{B}}\right)^{-1}\right]^{-1}$, and the ratio of the potential to thermal energy, $R$, is modified to

$$
R\left(\lambda_{\mathrm{D}, \Lambda}\right)=\frac{e \tilde{\Phi}\left(\lambda_{\mathrm{D}, \Lambda}\right)}{\ln \left(\frac{1+\Delta N_{e} / N_{\mathrm{D}}}{1-\Delta N_{i} / N_{\mathrm{D}}}\right) \frac{1}{2} k_{\mathrm{B}} T_{0}} \sim \frac{\kappa_{0} \cdot\left[\left(1-\frac{\Delta N_{i}}{N_{\mathrm{D}}}\right)^{-\frac{1}{\kappa_{0}+1}}-\left(1+\frac{\Delta N_{e}}{N_{\mathrm{D}}}\right)^{-\frac{1}{\kappa_{0}+1}}\right]}{\ln \left(\frac{1+\Delta N_{e} / N_{\mathrm{D}}}{1-\Delta N_{i} / N_{\mathrm{D}}}\right)} .
$$

The logarithm factor has been inserted so that $R\left(\lambda_{\mathrm{D}, \Lambda}\right)=1$ at thermal equilibrium $\left(\kappa_{0} \rightarrow \infty\right)$, and it is reduced to $\Delta N / N_{\mathrm{D}}$ for small values of $\Delta N / N_{\mathrm{D}}=\left(\Delta N_{e}+\Delta N_{i}\right) / N_{\mathrm{D}}$. Notion $\widetilde{\Phi}\left(\lambda_{\mathrm{D}, \Lambda}\right)$ is for the ' $p$-mean' (Livadiotis 2012) of the potential within a Debye sphere, with $p=-\left(\kappa_{0}+1\right)$, i.e.

$$
\bar{x}_{p} \equiv\left(\frac{n_{\infty}}{N_{\mathrm{D}}} \cdot \int_{0}^{\lambda_{\mathrm{D}, \Lambda}} x^{p} 4 \pi r^{2} d r\right)^{\frac{1}{p}}, \text { with } x \equiv 1+\frac{e \Phi(r)}{\kappa_{0} k_{\mathrm{B}} T_{i}}, \bar{x}_{p}=1+\frac{e \tilde{\Phi}\left(\lambda_{\mathrm{D}, \Lambda}\right)}{\kappa_{0} k_{\mathrm{B}} T_{i}} .
$$

Equation (E3) gives a functional dependence on $\kappa_{0}$ of the ratio $R$ at the Debye length, $R\left(\lambda_{\mathrm{D}, \Lambda}\right)$. This function must be equal to the functional of (12a),

$$
\begin{aligned}
R\left(\lambda_{\mathrm{D}, \Lambda} ; d\right)= & {\left[\frac{d}{2} \Gamma\left(\frac{d+1}{2}\right)\right]^{-1} \cdot \frac{\kappa_{0}}{\kappa_{0}+1} \cdot\left[\Lambda\left(\kappa_{0}\right) / \sqrt{\frac{\kappa_{0}}{\kappa_{0}+1}}\right]^{\frac{1+d}{2}} } \\
& \cdot \exp \left[-\Lambda\left(\kappa_{0}\right) / \sqrt{\frac{\kappa_{0}}{\kappa_{0}+1}}\right], d=1,2,3 .
\end{aligned}
$$

The equality of the two forms of the ratio $R\left(\lambda_{\mathrm{D}, 4}\right)$, (E3), (E5), leads to

$$
\begin{aligned}
F\left(\Lambda ; \kappa_{0}, \delta ; c\right) \equiv & \frac{\kappa_{0} \cdot\left[(1-\delta)^{-\frac{1}{\kappa_{0}+1}}-(1+\delta)^{-\frac{1}{\kappa_{0}+1}}\right]}{\ln [(1+\delta) /(1-\delta)]} \\
& -c \cdot \frac{\kappa_{0}}{\kappa_{0}+1} \cdot\left(\Lambda / \sqrt{\frac{\kappa_{0}}{\kappa_{0}+1}}\right)^{\frac{1+d}{2}} \cdot \exp \left(-\Lambda / \sqrt{\frac{\kappa_{0}}{\kappa_{0}+1}}\right) \rightarrow 0,
\end{aligned}
$$

where $\delta \equiv \Delta N_{e} / N_{\mathrm{D}} \cong \Delta N_{i} / N_{\mathrm{D}}$. The function $F$ is positive with a minimum, without any real roots $\Lambda$ for small values of $\kappa_{0}$. This minimum value of $F$ is its closest value to zero, and corresponds to $\Lambda\left(\kappa_{0}\right) \sim \frac{d+1}{2} \cdot \sqrt{\frac{\kappa_{0}}{\kappa_{0}+1}}$, that is again the modified Debye length, i.e. $\lambda_{\mathrm{D}, \Lambda} \sim \lambda_{\mathrm{D}, \mathrm{K}}$. 


\section{Appendix F. Statistical moments of the position}

Using the charge distribution in plasma,

$$
\rho(\mathbf{r})=e\left[n_{e}(\mathbf{r})-n_{i}(\mathbf{r})\right],
$$

with normalization given by the number of the excess electrons,

$$
\Delta N=\int_{r \in V}\left[n_{e}(\mathbf{r})-n_{i}(\mathbf{r})\right] d V, \text { or, } \Delta N e=\int_{\mathbf{r} \in V} \rho(\mathbf{r}) d V .
$$

Then, the average value of a positional function $f(r)$ is given by

$$
\langle f\rangle=\frac{1}{\Delta N e} \int_{\mathbf{r} \in V} f(\mathbf{r}) \rho(\mathbf{r}) d V,
$$

( $d V$ is the elementary volume). For small charge perturbations, the charge distribution is given by $(28 b)$,

$$
\rho(\mathbf{r})=e\left[n_{e}(\mathbf{r})-n_{i}(\mathbf{r})\right] \cong e n_{\infty} \cdot\left\langle\frac{\kappa_{0}+1}{\kappa_{0}}\right\rangle \cdot \frac{e \Phi(r)}{k_{\mathrm{B}} T_{0}},
$$

and the potential is given by (31),

$$
\frac{e \Phi(r)}{k_{\mathrm{B}} T_{0}}=\left[d \Gamma\left(\frac{d+1}{2}\right)\right]^{-1} \cdot \frac{\Delta N}{N_{\mathrm{D}}} \cdot\left\langle\frac{\kappa_{0}+1}{\kappa_{0}}\right\rangle^{-1} \cdot \frac{\exp \left(-r / \lambda_{\mathrm{D}}\right)}{\left(r / \lambda_{\mathrm{D}}\right)^{(d-1) / 2}},
$$

(written for any Debye length $\lambda_{\mathrm{D}}$ ), so that

$$
\rho(r) \propto \Phi(r) \propto \frac{\exp \left(-r / \lambda_{\mathrm{D}}\right)}{r^{(d-1) / 2}} .
$$

Hence, (F3) is written as

$$
\langle f\rangle \cong \frac{\int_{\mathbf{r} \in V} f(\mathbf{r}) \Phi(\mathbf{r}) d V}{\int_{\mathbf{r} \in V} \Phi(\mathbf{r}) d V} \cong \frac{\int_{\mathbf{r} \in V} f(\mathbf{r}) \frac{\exp \left(-r / \lambda_{\mathrm{D}}\right)}{r^{(d-1) / 2}} d V}{\int_{\mathbf{r} \in V} \frac{\exp \left(-r / \lambda_{\mathrm{D}}\right)}{r^{(d-1) / 2}} d V} .
$$

If the function $f$ is isotropic, i.e. $f(\mathbf{r})=f(r)$, then the elementary volume is $d V=$ $\left(B_{d} / d\right) L^{3-d} r^{d-1} d r$, and (F5a) becomes

$$
\langle f\rangle \cong \frac{\int_{0}^{\infty} f(r) \exp \left(-r / \lambda_{\mathrm{D}}\right) r^{(d-1) / 2} d r}{\int_{0}^{\infty} \exp \left(-r / \lambda_{\mathrm{D}}\right) r^{(d-1) / 2} d r} .
$$

As an example, let the $m$-statistical moment

$$
\left\langle r^{m}\right\rangle=\frac{1}{\Delta N} \int_{\mathbf{r} \in V}\left[n_{e}(\mathbf{r})-n_{i}(\mathbf{r})\right] r^{m} d V,
$$

which, according to (F5a), is

$$
\left\langle r^{m}\right\rangle \cong \frac{\int_{\mathbf{r} \in V} \Phi(\mathbf{r}) r^{m} d V}{\int_{\mathbf{r} \in V} \Phi(\mathbf{r}) d V}=\frac{\int_{0}^{\infty} \exp \left(-r / \lambda_{\mathrm{D}}\right) r^{m+(d-1) / 2} d r}{\int_{0}^{\infty} \exp \left(-r / \lambda_{\mathrm{D}}\right) r^{(d-1) / 2} d r}
$$

Hence,

$$
\left\langle r^{m}\right\rangle \cong \frac{\Gamma\left(m+\frac{d+1}{2}\right)}{\Gamma\left(\frac{d+1}{2}\right)} \cdot \lambda_{\mathrm{D}}^{m}
$$


The first and second moments

$$
\langle r\rangle \cong \frac{d+1}{2} \cdot \lambda_{\mathrm{D}} \text { and }\left\langle r^{2}\right\rangle \cong\left[\frac{d+1}{2}+1\right] \frac{d+1}{2} \cdot \lambda_{\mathrm{D}}^{2}
$$

lead to the positional variance

$$
\left\langle\Delta r^{2}\right\rangle=\left\langle r^{2}\right\rangle-\langle r\rangle^{2} \cong \frac{d+1}{2} \cdot \lambda_{\mathrm{D}}^{2},
$$

and positional standard deviation

$$
\sqrt{\left\langle\Delta r^{2}\right\rangle} \cong \sqrt{\frac{d+1}{2}} \cdot \lambda_{\mathrm{D}}
$$

Specifically for $d=1$, we have

$$
\sqrt{\left\langle\Delta x^{2}\right\rangle} \cong \lambda_{\mathrm{D}}
$$

The above equations are for small perturbations, and thus can be written in terms of the limit of $\Phi \rightarrow 0$, e.g.

$$
\lim _{\Phi \rightarrow 0}\left\langle\Delta r^{2}\right\rangle=\frac{d+1}{2} \cdot \lambda_{\mathrm{D}}^{2}, \lim _{\Phi \rightarrow 0}\left\langle\Delta x^{2}\right\rangle=\lambda_{\mathrm{D}}^{2}
$$

\section{REFERENCES}

Abe, S. 2001 General pseudoadditivity of composable entropy prescribed by the existence of equilibrium. Phys. Rev. E 63, 061105.

Bains, A. S., Tribeche, M. and Ng, C. S. 2013 Dust-acoustic wave modulation in the presence of q-nonextensive electrons and/or ions in dusty plasma. Astrophys. Space Sci. 343, 621-628.

Baumjohann, W. and Treumann, R. A. 2012 Basic Space Plasma Physics, Revised and Extended. London: Imperial College Press.

Bryant, D. A. 1996 Debye length in a kappa-distribution. J. Plasma Phys 56, 87-93.

Chen, F. F. 1974 Introduction to Plasma Physics. New York: Plenum.

Chotoo, K., et al. 2000 The suprathermal seed population for corotating interaction region ions at 1 AU deduced from composition and spectra of $\mathrm{H}+, \mathrm{He}++$, and $\mathrm{He}+$ observed by wind. $J$. Geophys. Res. 105, 23107.

Christon, S. P. 1987 A comparison of the Mercury and Earth magnetospheres: electron measurements and substorm time scales. Icarus 71, 448-471.

Collier, M. R. and Hamilton, D. C. 1995 The relationship between kappa and temperature in energetic ion spectra at Jupiter. Geophys. Res. Lett. 22, 303-306.

Dash, S. K. and Khuntia, S. R. 2010 Fundamentals of Electromagnetic Theory. New Delhi: PHI Learning, pp. 123.

Decker, R. B. and Krimigis, S. M. 2003 Voyager observations of low energy ions during solar cycle 23. Adv. Space Res. 32, 597-602.

Decker, R. B., Krimigis, S. M., Roelof, E. C., Hill, M. E., Armstrong, T. P., Gloeckler, G., Hamilton, D. G. and Lanzerotti, L. J. 2005 Voyager 1 in the foreshock, termination shock, and heliosheath. Science 309, 2020-2024.

Dialynas, K., Krimigis, S. M., Mitchell, D. G., Hamilton, D. C., Krupp, N. and Brandt, P. C. 2009 Energetic ion spectral characteristics in the Saturnian magnetosphere using Cassini/MIMI measurements. J. Geophys. Res. 114, A01212.

Eslami, P., Mottaghizadeh, M. and Pakzad, H. R. 2011 Nonplanar dust acoustic solitary waves in dusty plasmas with ions and electrons following a q-nonextensive distribution. Phys. Plasmas 18, 102303.

Fahlen, J. E., Winjum, B. J., Grismayer, T. and Mori, W. B. 2011 Transverse plasma-wave localization in multiple dimensions. Phys. Rev. E 83, 045401.

Gougam, L. A. and Tribeche, M. 2011 Debye shielding in a nonextensive plasma. Phys. Plasmas 18, 062102.

Grabbe, C. 2000 Generation of broadband electrostatic waves in Earth's magnetotail. Phys. Rev. Lett. 84, 3614. 
Heerikhuisen, J., Pogorelov, N. V., Florinski, V., Zank, G. P. and le Roux, J. A. 2008 The effects of a k-distribution in the heliosheath on the global heliosphere and ENA flux at 1 AU. Astrophys. J. 682, 679-689.

Hellberg, M. A., Mace, R. L., Baluku, T. K., Kourakis, I. and Saini, N. S. 2009 Comment on 'Mathematical and physical aspects of Kappa velocity distribution' [Phys. Plasmas 14, 110702 (2007)]. Phys. Plasmas 16, 094701.

Kallenrode, M.-B. 2004, Space Physics: An Introduction to Plasmas and Particles in the Heliosphere and Magnetospheres. Berlin: Springer-Verlag.

Kourakis, I., Sultana, S. and Hellberg, M. A. 2012 Dynamical characteristics of solitary waves, shocks and envelope modes in kappa-distributed non-thermal plasmas: an overview. Plasma Phys. Control. Fusion 54, 124001.

Krall, N. A. and Trivelpiece, A. W. 1973 Principles of Plasma Physics, McGraw-Hill: Kogakusha, LTD.

Le Roux, J. A., Webb, G. M., Shalchi, A. and Zank, G. P. 2010 A generalized nonlinear guiding center theory for the collisionless anomalous perpendicular diffusion of cosmic rays. Astrophys. $J$. 716, 671-692.

Leubner, M. P. 2004 Fundamental issues on kappa-distributions in space plasmas and interplanetary proton distributions. Phys. Plasmas 11, 1306-1308.

Livadiotis, G. 2009 Approach on Tsallis statistical interpretation of hydrogen-atom by adopting the generalized radial distribution function. J. Math. Chem. 45, 930-939.

Livadiotis, G. 2012 Expectation values and variance based on $L_{p}$-norms. Entropy 14, 2375.

Livadiotis, G. and McComas, D. J. 2009 Beyond kappa distributions: exploiting Tsallis statistical mechanics in space plasmas. J. Geophys. Res. 114, A11105.

Livadiotis, G. and McComas, D. J. 2010a Exploring transitions of space plasmas out of equilibrium. Astrophys. J. 714, 971-987.

Livadiotis, G. and McComas, D. J. 2010b Measure of the departure of the q-metastable stationary states from equilibrium. Phys. Scr. 82, 035003.

Livadiotis, G. and McComas, D. J. 2010c Non-equilibrium stationary states in the heliosphere: the influence of pick-up ions. AIP Conf. Proc. 1302, 70-76.

Livadiotis, G. and McComas, D. J. 2011a The influence of pick-up ions on space plasma distributions. Astrophys. J. 738, 64.

Livadiotis, G. and McComas, D. J. 2011b Invariant kappa distribution in space plasmas out of equilibrium. Astrophys. J. 741, 88.

Livadiotis, G. and McComas, D. J. 2012 Non-equilibrium thermodynamic processes: space plasmas and the inner heliosheath. Astrophys. J. 749, 11.

Livadiotis, G. and McComas, D. J. 2013a Evidence of large scale phase space quantization in plasmas. Entropy 15, 1116-1132.

Livadiotis, G. and McComas, D. J. 2013b Understanding kappa distributions: a toolbox for space science and astrophysics. Space Sci. Res. 175, 183-214.

Livadiotis, G. and McComas, D. J. 2013c Fitting method based on correlation maximization: applications in space physics. J. Geophys. Res. A118, 2863-2875.

Livadiotis, G. and McComas, D. J. 2013d Near-equilibrium heliosphere - far-equilibrium heliosheath. AIP Conf. Proc. 1539, 344-347.

Livadiotis, G. and McComas, D. J. 2013e Large-scale quantization in space plasmas. Summary and applications. Proc. Astron. Soc. Pacific (In Press).

Livadiotis, G., McComas, D. J., Dayeh, M. A., Funsten, H. O. and Schwadron, N. A. 2011 First sky map of the inner heliosheath temperature using IBEX spectra. Astrophys. J. 734, 1.

Livadiotis, G., McComas, D. J., Randol, B. M., Funsten, H. O., Moebius, E. S., Schwadron, N. A., Dayeh, M. A., Zank, G. P. and Frisch, P. C. 2012 Pick-up ion distributions and their influence on energetic neutral atom spectral curvature. Astrophys. J. 751, 64.

Livadiotis, G., McComas, D. J., Schwadron, N. A., Funsten, H. O. and Fuselier, S. A. 2013 Pressure of the proton plasma in the inner heliosheath. Astrophys. J. 762, 134.

Maksimovic, M., et al. 2005 Radial evolution of the electron distribution functions in the fast solar wind between 0.3 and 1.5 AU. J. Geophys. Res. 110, A09104.

Mann, G., Classen, H. T., Keppler, E. and Roelof, E. C. 2002 On electron acceleration at CIR related shock waves. Astron. Astrophys. 391, 749-756. 
Mauk, B. H., Mitchell, D. G., McEntire, R. W., Paranicas, C. P., Roelof, E. C., Williams, D. J., Krimigis, S. M. and Lagg, A. 2004 Energetic ion characteristics and neutral gas interactions in Jupiter's magnetosphere. J. Geophys. Res. 109, A09S12.

Milovanov, A. V. and Zelenyi, L. M. 2000 Functional background of the Tsallis entropy: 'coarsegrained' systems and 'kappa' distribution functions. Nonlinear Process. Geophys. 7, 211-221.

Milovanov, A. V. and Zelenyi, L. M. 2001 'Strange' Fermi processes and power-law nonthermal tails from a self-consistent fractional kinetic equation. Phys. Rev. E 64, 052101.

Montgomery, D. C. and Tidman, D. A. 1964 Plasma Kinetic Theory. New York: McGraw-Hill.

Ogasawara, K., Angelopoulos, V., Dayeh, M. A., Fuselier, S. A., Livadiotis, G., McComas, D. J. and McFadden, J. P. 2013 Diagnosing dayside magnetosheath using energetic neutral atoms: IBEX and THEMIS observations. J. Geophys. Res. A118, 3126-3137.

Pierrard, V. and Lazar, M. 2010 Kappa distributions: theory and applications in space plasmas. Sol. Phys. 267, 153-174.

Raadu, M. A. and Shafiq, M. 2007 Test charge response for a dusty plasma with both grain size distribution and dynamical charging. Phys. Plasmas 14, 012105.

Rubab, N. and Murtaza, G. 2006 Debye length in non-Maxwellian plasmas. Phys. Scr. 74, 145.

Saberian, E. and Esfandyari-Kalejahi, A. 2013 Langmuir oscillations in a nonextensive electronpositron plasma. Phys. Rev. E 87, 053112.

Saito, S., Forme, F. R. E., Buchert, S. C., Nozawa, S. and Fujii, R. 2000 Effects of a kappa distribution function of electrons on incoherent scatter spectra. Ann. Geophys. 18, 1216-1223.

Schippers, P., et al. 2008 Multi-instrument analysis of electron populations in Saturn's magnetosphere. J. Geophys. Res. 113, A07208.

Treumann, R. A. 1999 Generalized-Lorentzian thermodynamics. Phys. Scripta. 59, 204-214.

Treumann, R. A. and Jaroschek, C. H. 2008 Gibbsian theory of power-law distributions. Phys. Rev. Lett. 100, 155005.

Treumann, R. A., Jaroschek, C. H. and Scholer, M. 2004 Stationary plasma states far from equilibrium. Phys. Plasmas 11, 1317.

Tribeche, M., Mayout, S. and Amour, R. 2009 Effect of ion suprathermality on arbitrary amplitude dust acoustic waves in a charge varying dusty plasma. Phys. Plasmas 16, 043706.

Tsallis, C. 1988 Possible generalization of Boltzmann-Gibbs statistics. J. Stat. Phys. 52, 479-487.

Tsallis, C. 2009 Introduction to Non-extensive Statistical Mechanics: Approaching a Complex World. New York: Springer.

Tsallis, C., Mendes, R. S. and Plastino, A. R. 1998 The role of constraints within generalized nonextensive statistics. Physica A 261, 534-554.

Vasyliũnas, V. M. 1968 A survey of low-energy electrons in the evening sector of the magnetosphere with OGO 1 and OGO 3. J. Geophys. Res. 73, 2839-2884.

Yoon, P. H. 2012 Electron kappa distribution and steady-state Langmuir turbulence. Plasma Phys. 19, 052301.

Yoon, P. H., Rhee, T. and Ryu, C. M. 2006 Self-consistent formation of electron $\kappa$ distribution. $J$. Geophys. Res. 111, A09106.

Yoon, P. H., Ziebell, L. F., Gaelzer, R., Lin, R. P. and Wang, L. 2012 Langmuir turbulence and suprathermal electrons. Space Sci. Rev. 173, 459-489.

Zank, G. P., Heerikhuisen, J., Pogorelov, N. V., Burrows, R. and McComas, D. J. 2010 Microstructure of the heliospheric termination shock: implications for energetic neutral atom observations. Astrophys. J. 708, 1092. 